\title{
Reframing Psychiatry for Precision Medicine
}

\author{
Elizabeth B. Torres ${ }^{1,2,3}$ \\ 1 Department of Psychology, Rutgers University, New Brunswick, NJ 08854, USA; \\ ebtorres@psychology.rutgers.edu; Tel.: +1-(011)-858-445-8909; Fax: +1-(011)-732-445-2987 \\ 2 Center for Cognitive Science (RUCCS), Rutgers University, New Brunswick, NJ 08854, USA \\ 3 Computer Science, Center for Biomedicine Imaging and Modelling (CBIM), Rutgers University, \\ New Brunswick, NJ 08854, USA
}

Received: 19 July 2020; Accepted: 16 September 2020; Published: 25 September 2020

\begin{abstract}
The art of observing and describing behaviors has driven diagnosis and informed basic science in psychiatry. In recent times, studies of mental illness are focused on understanding the brain's neurobiology but there is a paucity of information on the potential contributions from peripheral activity to mental health. In precision medicine, this common practice leaves a gap between bodily behaviors and genomics that we here propose to address with a new layer of inquiry that includes gene expression on tissues inclusive of brain, heart, muscle-skeletal and organs for vital bodily functions. We interrogate gene expression on human tissue as a function of disease-associated genes. By removing genes linked to disease from the typical human set, and recomputing gene expression on the tissues, we can compare the outcomes across mental illnesses, well-known neurological conditions, and non-neurological conditions. We find that major neuropsychiatric conditions that are behaviorally defined today (e.g., autism, schizophrenia, and depression) through DSM-observation criteria have strong convergence with well-known neurological conditions (e.g., ataxias and Parkinson's disease), but less overlap with non-neurological conditions. Surprisingly, tissues majorly involved in the central control, coordination, adaptation and learning of movements, emotion and memory are maximally affected in psychiatric diagnoses along with peripheral heart and muscle-skeletal tissues. Our results underscore the importance of considering both the brain-body connection and the contributions of the peripheral nervous systems to mental health.
\end{abstract}

Keywords: autism; schizophrenia; mental depression; ataxia; fragileX; Parkinson's disease; mitochondria; gene expression; tissues; neurological disorders; nervous systems disorders

\section{Introduction}

Modern medicine is at an inflexion point [1], whereby advances in computational methods, wearable sensing technology and open access to Big Data are reshaping the ways in which we inform basic science and rapidly translate our knowledge to actionable treatments. Psychiatry is one of those medical fields that is rapidly evolving, while adapting traditional models to help advance the main goal of helping patients improve their quality of life. Along those lines, computational psychiatry [2], a nascent subfield within psychiatry, is merging methods from Computational Neuroscience with clinical approaches through successful collaborations. These new developments are bound to open new frontiers in therapeutic treatments. Further, as part of a more general effort in the medical field, precision medicine (PM) [1] has emerged as a new platform to combine expertise from multiple layers of the knowledge network in order to ultimately design personalized targeted treatments (Figure 1A). Integrating the personalized concept of PM with the new advances in computational psychiatry could give us a new way to approach mental illness and help patients cope with lifelong changing needs. 
A

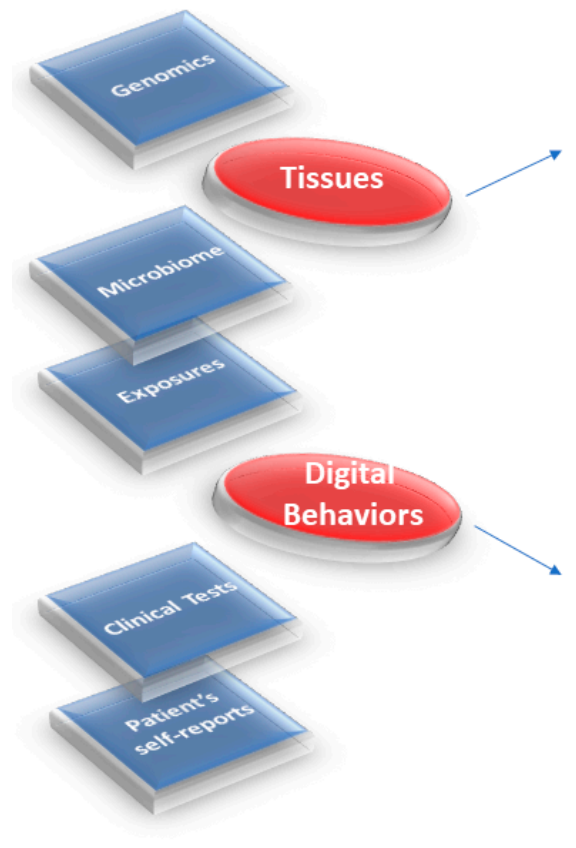

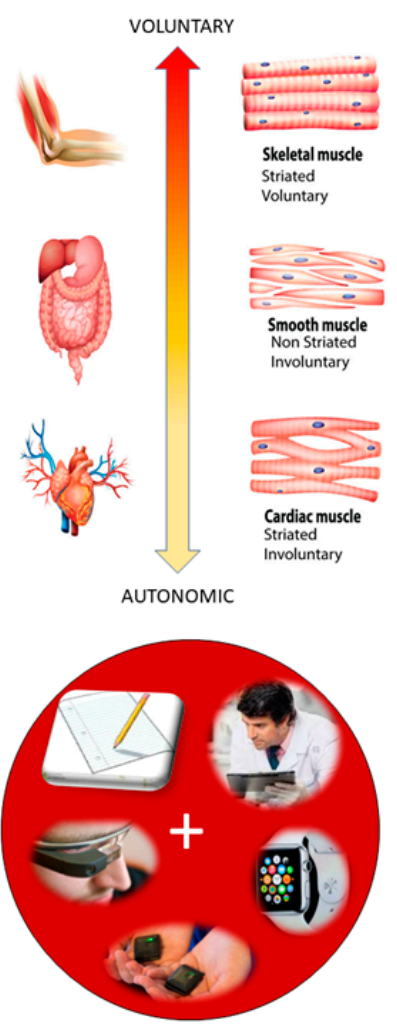

Figure 1. Roadmap to implement the precision medicine model for diagnoses and treatments of mental illnesses. (A) PM's interconnected knowledge network can contribute information about the individual's medical history, behaviors, environment, microbiome, and genetic makeup. Importantly, the new proposed layer of digitized behaviors leveraging the wearable biosensors revolution can transform medicine by creating truly personalized assessments. Additionally, the layer of behaviors can be connected to nervous system functioning via fundamental levels of neuromotor control that span along a phylogenetically orderly taxonomy. (B) This proposed taxonomy is based on levels of maturation in autonomous neuromotor control, linked to three fundamental muscle types: autonomic (by cardiac muscles), involuntary (by smooth muscles) and voluntary (by skeletal muscles). By linking the fundamental muscle types to the levels of control in the nervous systems, digital behaviors can then be mapped to bodily autonomy, bodily autonomy mapped to muscle types and muscle types mapped to genes/proteins. Any measure of treatment effectiveness for mental illnesses can then map back to improvements in observable behaviors embedded in activities of daily social life.

The task ahead is challenging because there is no proper roadmap to connect the layers of the knowledge network in PM and produce personalized diagnoses and measures of treatment outcomes that truly separate disease progression from treatment effectiveness according to age and development. Part of the problem is that most brain science has focused on experimental assays and methods that curtail natural movements. As such, our knowledge about the dynamics of natural behaviors is very limited, particularly in reference to those aspects of behavior that remain hidden to the naked eye of the clinician trained to observe specific expected behavioral landmarks of a psychiatric disorder conceived exclusively as a mental illness. In so doing, the clinically trained eye may miss important information that is perhaps common across different disorders of the nervous systems and rather relevant to help improve the patient's quality of life. For example, motor coordination and volitional control are critical ingredients of autonomy in any natural behavior underlying activities of daily living. Yet, these are not considered part of the diagnostics criteria for mental illnesses such as autism, schizophrenia and depression, as per the Diagnostics Statistical Manual (DSM-5) [3] (and see Supplementary Materials).

Research on the underlying neurobiology of mental illnesses has revealed their associated genetics [4] and/or helped characterize patterns of brain activity in response to external stimuli [5] 
(while curtailing naturalistic bodily motions to avoid instrumentation artifacts in imaging data or in EEG, MEG, etc.). This central approach to brain science has left us with a paucity of information about the possible contributions to mental illness from the peripheral nervous systems, and from vital organs important for autonomous living. The peripheral activity, however, continuously feeds back to the brain via afferent (body-to-brain) channels and is, in turn, dynamically updated through efferent (brain-to-body) activity, self-generated by the system itself. This recursive loop, whereby re-entrant information that is partly self-produced by the organism and partly influenced by external environmental conditions, would provide important clues about truly evolving dynamics and stochastic (variability) across all-natural behaviors. Approaching the problem through this lens could bring a new quantifiable layer of granularity to basic research. This would include the design of age-appropriate metrics reflecting the development of the organism as it ages and as it copes with a disorder $[6,7]$. The micro- and macro-motion data from the nervous systems biorhythms is the low hanging fruit that we can easily attain by leveraging the wearable sensors revolution. Further, because these quantifiable digitized activities and signals therein are partly self-generated, self-monitored and self-corrected by and within the nervous systems, this quantitative approach has the potential to take us from a purely correlational science to a science that is based on causal relations between nervous system activities and external/contextual stimuli. In this sense, the new proposed approach to mental illness is amenable to intervene and modify the system with well-informed, near-optimal means capable of improving its performance.

Micro- and macro-motions that underly all aspects of human behavior depend on the intactness of fundamental tissues, many of which have already been characterized in genomics according to cell types [8]. Here, we propose to combine micro-level underlying aspects of behavior with the current genomics knowledge to inform psychiatry of possible ways to improve quantification of nervous system activities. Ultimately, we seek to compile this information to help build accommodations and support for the patient population, while reconceptualizing mental illness as a physically quantifiable disorder of the nervous systems.

The nervous systems already offer a taxonomy of function and control that is phylogenetically ordered and well organized along several axes. Some of these axes are accessible today with non-invasive means and, as such, we can obtain signals and build computational models to understand mechanisms and translate them to actionable societal solutions. One possible orderly structure is suggested in Figure 1B, where we propose to map levels of neuromotor control (voluntary, involuntary, and autonomic) to fundamental types of muscles (skeletal, smooth, and cardiac) linked to commonly sampled tissues in genomic datasets. Combining information about gene expression on tissues that involve key components of the central nervous systems (the brain and the spinal cord), key organs for vital bodily functions (including smooth muscle lining internal organs), muscle-skeletal tissues and nerves, and cardiac tissues (for autonomic heart functioning), we explore the effects of removing disease-associated genes, on the overall remaining genome expression on these tissues. As a first step in this exercise, we reasoned that the genes associated with a given disorder ought to be important in the functioning of certain systems, which in turn depend on certain tissues. We also reasoned that such stochastic variations and combinations could be measured relative to the presence of all genes and to the absence of genes across neurological or non-neurological conditions.

What is the tissue distribution of gene expression in neuropsychiatric disorders such as autism, schizophrenia, and depression in relation to well-characterized neurological conditions? Is there convergence in the remaining gene expression on the tissues upon removal of the genes associated with that disease? Furthermore, how would the gene expression change across the tissues in non-neurological conditions such as various forms of cancer, immunodeficiencies, endocrine system deficiencies and so forth? How would it change in acquired disorders such as Post Traumatic Stress Syndrome (PTSD), currently diagnosed through observation?

Take autism for example. Autism is an umbrella term for a very heterogeneous set of neurodevelopmental disorders, but no gold-standard criteria include core neurological symptoms 
that could help us create early accommodations and support for the nascent nervous systems of the infant (during pre-cognitive stages of neurodevelopment). The rule of thumb is to assume that the child has odd, socially inappropriate behaviors and that they should be modified through operant and cognitive conditioning techniques-often translated from lab animals to human babies, without any type of collaboration with other fields studying infant development. Current methods of diagnoses and treatments in autism are not based on normative neurodevelopmental data charts to understand age-dependent departures from typical neurodevelopment. Without any systematic way to build age-appropriate metrics in order to capture highly non-linear, stochastic patterns and rates of change in the (rather accelerated) infant neurodevelopment, entire generations of infants, children and adolescents have been exposed to such means of behavioral treatments and no information can tie these back to the underlying genomic pool of this population.

In schizophrenia, delusions, avolition and catatonia are at the core of the disorder, but as in autism above, no criteria in the DSM highlight the profound somatic sensory-motor issues that have been found in patients [9] — even without the use of psychotropic medications known to alter motility. Interestingly, historical accounts of psychiatry (in pre-Freudian times) show the reliance on motor aspects of the behaviors that defined several mental illness from a neurological perspective [10].

Depression is also currently treated purely as a mental illness, but it may be important to understand potential contributions to various forms of depression, from the peripheral nervous systems and from the body in general. Genetic information may give us a way to link tissues affected in these neuropsychiatric conditions with those affected in neurological conditions, for which treatments and interventions of various forms may be effective. These may be in the form of drugs, or in the form of physical, mindfulness and occupational therapies aimed at helping support the person's bodily autonomy and overall increase the chances for independent living.

We here offer a new lens to help balance psychiatric with neurological criteria derived from genomic information specific to each disorder. In a first (crude) step of many to come, we start by comparing well-known neuropsychiatric and neurological conditions, the results from eliminating the genes associated with each disorder and quantifying the degree of convergence in the maximally affected tissues, in relation to those resulting from eliminating the genes associated with non-neurological conditions. We focus our discussion on possible ways to continue this path of inquiry and highlight current caveats for future improved iterations of the proposed methods.

\section{Materials and Methods}

We combine the datasets from genes associated with mental illnesses with well-known neurological disorders and with illnesses that are not directly associated with the nervous systems. We also include genes associated with manifestations of acquired Post-Traumatic Stress Syndrome Disorder (PTSD). Among mental illnesses defined by the DSM-5, we include autism, schizophrenia and mental depression of different types, (e.g., general, bipolar and unipolar). Among neurological conditions, we include ataxias (e.g., cerebellar, spinocerebellar, progressive, and gait) and Parkinson's disease. Among non-neurological disorders, we include colon cancer, breast cancer, diabetes, congenital heart disease, hematologic neoplasm, and various autoimmune disorders (lupus systemic erythematosus, psoriasis, and irritable bowel syndrome).

We use the genes, gene expression, and tissues from the Genotype-Tissue Expression project, GTEx pPortal human RNA-seq (Transcripts Per Million (TPM), see Appendix A for note in TPM) as reference specifically using the files denoted in Appendix B. In autism, we use the gene scoring module of the Simons Foundation Autism Research Initiative (SFARI) scored according to evidence from the literature. We also use ataxia genes, the $\mathrm{X}$ genes and the FX genes taken from various literature reviews $[11,12]$. Furthermore, we use genes associated with mitochondrial disorders [13] and genes identified in Parkinson's disease, taken from [14-19]. Besides the autism SFARI genes and the genes reported in literature reviews, we take the genes associated with autism, schizophrenia and depression reported in https://www.disgenet.org/home/ along with other genes from the above-mentioned non-neurological 
disorders. The latter will inform us of fundamental differences in gene expression between these diseases and those which affect neuromotor control and basic functioning, as mediated by interactions between the brain and the peripheral nervous systems (including the autonomic nervous system).

The SFARI autism categories that we used were those reported as of 03-04-2020. Quoting from their site:

- CATEGORY 1 Genes in this category are all found on the SPARK gene list. Each of these genes has been clearly implicated in Autism Spectrum Disorders, ASD-typically by the presence of at least three de novo likely-gene-disrupting mutations being reported in the literature-and such mutations identified in the sequencing of the SPARK cohort are typically returned to the participants. Some of these genes meet the most rigorous threshold of genome-wide significance; all at least meet a threshold false discovery rate of $<0.1$.

- CATEGORY 2 Genes with two reported de novo likely-gene-disrupting mutations. A gene uniquely implicated by a genome-wide association study, either reaching genome-wide significance or, if not, consistently replicated and accompanied by evidence that the risk variant has a functional effect.

- CATEGORY 3 Genes with a single reported de novo likely-gene-disrupting mutation. Evidence from a significant but unreplicated association study, or a series of rare inherited mutations for which there is not a rigorous statistical comparison with controls.

- SYNDROMIC The syndromic category includes mutations that are associated with a substantial degree of increased risk and consistently linked to additional characteristics not required for an ASD diagnosis. If there is independent evidence implicating a gene in idiopathic ASD, it will be listed as "\#S" (e.g., 2S, 3S). If there is no such independent evidence, the gene will be listed simply as "S".

The GTEx dataset is as the 06-05-2017 v8 release. For every gene in autism, ataxia, X, FX, mitochondrial diseases, Parkinson's disease, and the non-neurological diseases, we first confirmed the presence of the gene in the GTEx dataset and then incorporated it into the analyses.

The genes from the DisGeNet portal were found by interrogation of their dataset under disease type and saving the outcome to excel files containing all pertinent information. All sample files used in our analyses are provided in Supplementary Materials.

\subsection{Count Normalization}

The GTEx matrix of RNA-seq genes along the rows $(56,146) \times$ the tissues $(54)$ along the columns was transposed $(54 \times 56,246)$, such that we expressed each tissue as a function of the gene expression denoted by the count (TPM). Each individual count value was then normalized using Equation (1).

$$
\text { Normalized Count }=\frac{\text { count }_{i}}{\text { count }_{i}+\frac{\text { Avrg }_{\text {Global }} \text { Count }}{\operatorname{Max}_{\text {Global }} \text { Count }}}
$$

Here, count $t_{i}$ is the count value of the gene $\mathrm{i}_{\mathrm{i}}$, Avrg $_{\text {Global }}$ Count is the overall average of the matrix of values taken along the columns and the rows. Max ${ }_{\text {Global }}$ Count is the maximum count value, also taken globally across the matrix values. Figure 2 shows the original count numbers (Figure 2A) and the normalized version (coined micro-movement spikes (MMS)) in Figure 2B. Figure 2C shows the MMS derived from the fluctuations in counts normalized by Equation (1), while Figure 2D shows the histograms of the peaks (marked in red dots) for different tissues and genes scored by the SFARI. 
A

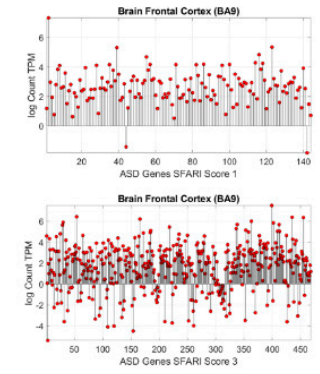

C

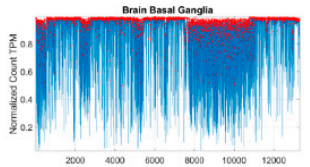

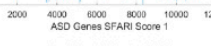

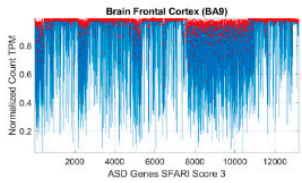

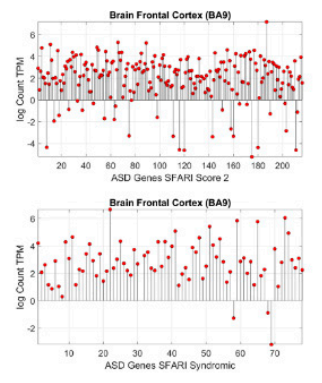

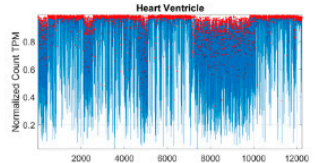

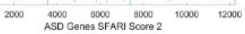

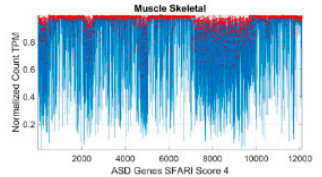

B

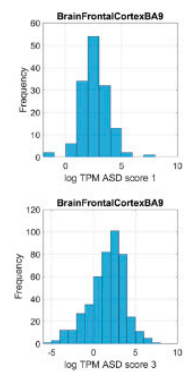

D

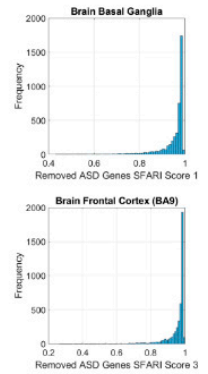

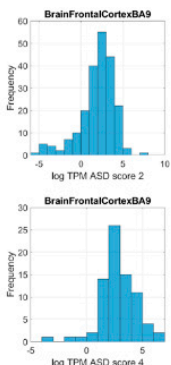

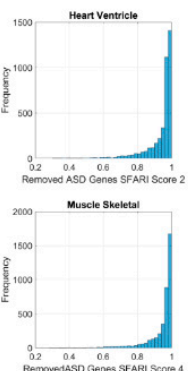

Figure 2. Analytical methods. (A) Sample raw data consisting of the log count (TPM) for different scored genes expressed in the brain frontal cortex (Brodmann Area 9). (B) Histograms of the log count TPM for each case in (A). (C) Upon removal of the Simons Foundation Autism Research Initiative (SFARI) autism genes, we obtain micro-fluctuation spikes in the normalized count, with deviations taken relative to empirically estimated mean, global averaged count, and global maximal count in Equation (1). (D) Histograms of the normalized micro-fluctuation spikes.

\subsection{Gene Removal}

For each of the disorders of interest in Appendix B Table A1 (mental illness and neurological) and Table A2 (non-neurological), we remove the genes associated with each condition from the human GTEx dataset. These disease-gene associations are as reported in the various databases (the SFARI, DisGeNet https://www.disgenet.org/home/ and the literature meta reviews). We then treat the resulting count series as a random process. We use the exponential distribution to characterize it and to assess the differential expression across the tissues relative to non-removal in the original human genome.

The question that we ask is: given the known neurological phenotypes, is there convergence between the most-affected tissues upon associated gene removal and the changes in the tissues that will be obtained by the removal of genes associated with mental illnesses? Furthermore, is there convergence with the outcome from removing the genes associated with non-neurological illnesses? Appendix B Tables A1 and A2 show the number of genes removed in each respective case as well as the source of the reported genes associated with each condition/disease.

\subsection{Stochastic Analyses}

Since the count values for each tissue can be conceived as a random series of numbers, we use maximum likelihood estimation (MLE) to model the numbers representing the counts, as generated by the exponential distribution using Equation (2)

$$
y=\lambda e^{-\lambda x}
$$

Here, $x$ represents the normalized count value (as per Equation (1)) and $y$ represents the value from the exponential distribution. We seek the value of the rate parameter $\lambda$ to model this random counting process, which we use to represent the gene expression in the tissue. To that end, we estimate the likelihood $L\left(\lambda \mid x_{1}, x_{2}, \ldots, x_{n}\right)$ where the series of counts $x_{i}$, with $i$ ranging from 1 to $n$, represent the normalized counts (according to Equation (1)) across all genes for one tissue. Appendix B shows the 
steps to find $\lambda$. Further, this is computed for each of the 54 tissues. We then rank the departure of $\lambda$ (resulting from gene removal) from the $\lambda$ obtained for the full human genome (see below). This is explained in Figure 3.

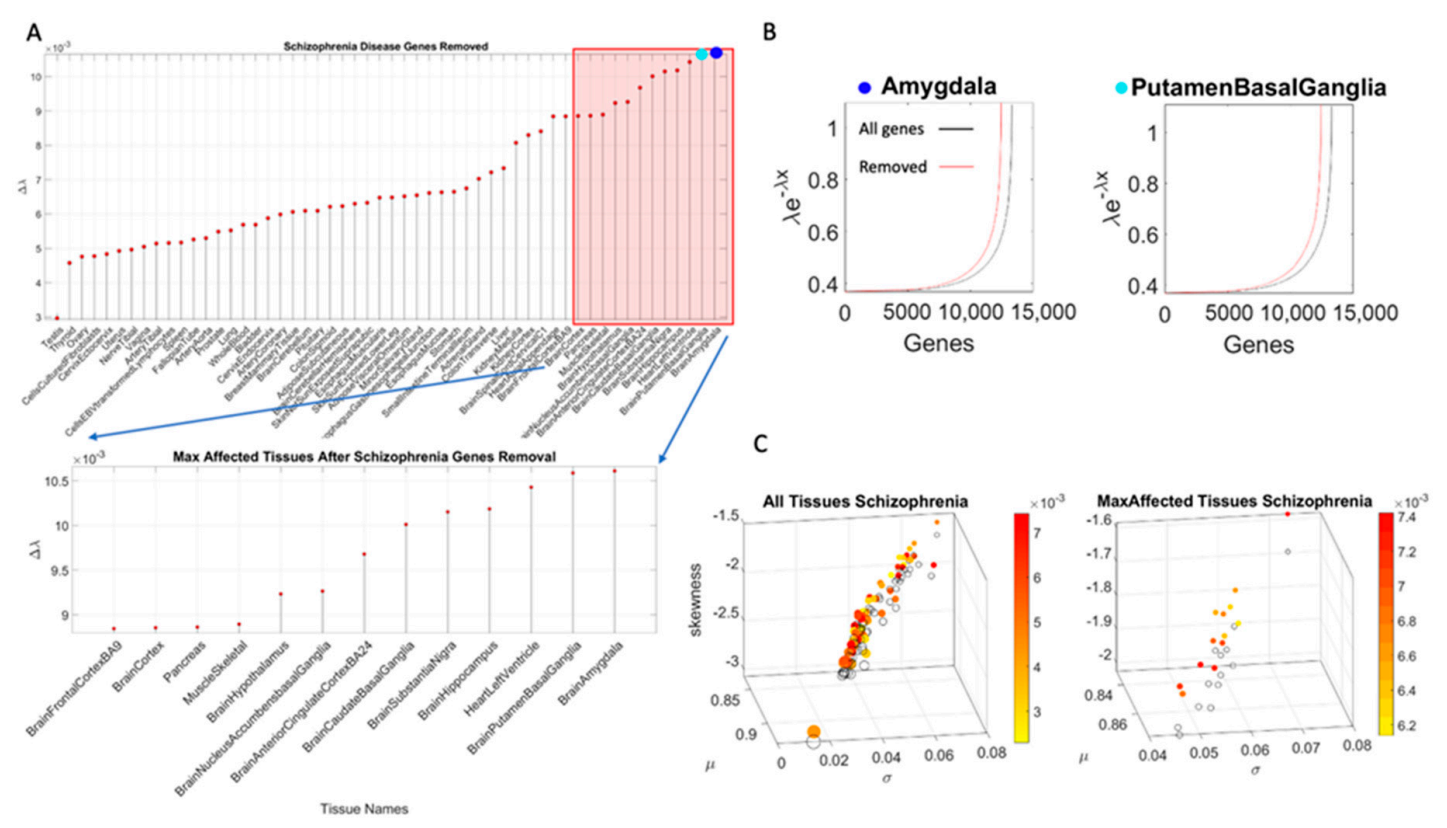

Figure 3. Sample metrics used for the stochastic analyses of a data sample (using 2697 schizophrenia-associated genes reported in DisGenNet portal and in the literature). (A) Effect of removing the schizophrenia genes from the GTEx human genome set expressed across 54 tissues. Tissues are sorted in ascending order, by the absolute difference $\Delta \lambda$ between gene expression on the 54 tissues before and after removal. The red square highlights the top 13 median-ranked tissues shown in the panel below and the dark and light blue circles mark the top two tissues affected (the brain amygdala and brain putamen in the basal ganglia. (B) The exponential distribution curve is fit to the sorted normalized count representing the gene's expression in TMP on the top median-ranked affected tissues (as in Figure 2C, taking the peaks highlighted in red and fitting the exponential distribution to the frequency histogram, as in Figure 2D) before (black line) and after (red line) the removal of the genes associated with the disease. The absolute value difference between the curves is the $\Delta \lambda$ used to rank the tissues by the effect size. (C) The fitting of the gamma distribution yields the shape and scale parameters used to compute the gamma moments. The axes represent the mean, the variance, and the skewness of the distribution of the normalized values and the color map represents the Earth Mover's Distance values measuring the difference between the resulting exponential frequency histograms in (B). The size of the circle is proportional to the kurtosis and the color-filled circles represent the tissue (54 in the left panel) with the original gene expression from GTEx (our reference template) vs. the open circles representing the stochastic shift, i.e., upon the removal of the genes associated with the disease in DisGenNet. The right panel contains the top-ranked tissues (13) according to the median values of the $\Delta \lambda$.

\subsection{Stochastic Analyses-Visualization of Change Relative to the Normative Data of the Full Human Genome}

Using MLE, we also obtain for each of the 54 tissues, the frequency histograms of the normalized counts across all genes and fit the continuous gamma family of probability distributions with shape $(a)$ and scale $(b)$ values in order to obtain the gamma moments and plot them on a parameter space. We do this to visualize the spread of the tissues and their shift upon gene removal. To that end, we plot the mean, the variance, and the skewness across the $x, y$ and $z$ axes, respectively. We plot the size of the marker representing the tissue proportional to the kurtosis value, and we color the marker based on the change relative to the original genome count (i.e., containing all the genes, without removal). 
To measure the stochastic shift between the tissues from the full genome and those upon removal of the genes identified with each known neurological condition, we take the absolute difference between the MLE $\lambda$ for the full GTEx genome and that for the genome upon removal of the genes associated with each condition, disorder or disease, as shown in Figure 3.

We median rank the $\Delta \lambda$ for each tissue, sorting $\Delta \lambda$ in ascending order across the 54 tissues. Then we create four median-ranked blocks and plot the maximally affected block of tissues (Figure 3A). The highest-ranked group is then compared across all conditions-mental illness vs. those neurologically defined vs. those non-neurologically defined. We annotate the neurological functions that such tissues are known to maximally disrupt. Further, we determine whether there is convergence between the tissue outcome in mental illnesses, upon removing the associated genes from the human genome-tissue model, and the outcome upon removing those genes tied to the other known disorders of the nervous systems. We repeat this interrogation process using the genes associated with disorders in the DisGeNet portal, including autism, schizophrenia, depression, the neurological disorders in Appendix B Table A1, and the non-neurological disorders in Appendix B Table A2.

To assess tissue outcome upon removal of genes associated with various non-neurological diseases, we follow these procedures and compare these to the above results. These diseases include colon cancer, breast cancer, psoriasis, diabetes, congenital heart disease, hematologic neoplast and systemic lupus. Appendix B Table A2 describes the number of genes associated with each of these diseases and the sources.

We also examine other mental illnesses described by the DSM. These include schizophrenia, depression, unipolar depression, and bipolar depression. We determine whether there are tissues that overlap with those affected in the neurological disorders. Lastly, we examine mitochondria-related disorders and PTSD using these methods. We reasoned that these may be disorders that have potentially affected tissues across a broader range of functions, including those from the brain and other bodily organs.

\section{Results}

\subsection{Autism, Ataxia and FX Have Convergence in Maximally Affected Tissues by the Removal of Associated Genes}

The maximally affected tissues upon gene removal, according genes stochastic expression (count in Transcripts Per Million (TPM)), are depicted in Appendix B Table A3. Tissue gene expression was modelled by the exponential distribution $y=\lambda e^{-\lambda x}$, with $x$ as the gene combination expressed in the tissues, and $\lambda$ as the exponential rate parameter. The $\Delta \lambda$ between the neurotypical template case from the GTEx portal (containing all genes) and the modeled disorder case (upon removal of the SFARI genes) provides a sense of the departure from the normative case. This difference, taken for the removal of the SFARI genes, is depicted in Figure 4A, with samples of maximally affected tissues in Figure 4B that are known to be critical for motor control, regulation, adaptation/learning, and coordination.

The $\Delta \lambda$ median ranking quantified the difference between neurotypical tissue gene expression vs. tissue gene expression upon removal of the genes corresponding to the disorders in question, with four groups ordered by the size of $\Delta \lambda$. This $\lambda$ quantity was first obtained relative to the neurotypical population tissues, i.e., including all the counts (gene expression) from all genes, in order to model an exponential process. The computation of $\lambda$ using maximum likelihood estimation (MLE) is explained in Appendix A. It does not assume any order of the counts, but rather seeks to identify the resulting $\lambda$ for each tissue, treating the gene count (expression) as a random, memoryless stochastic process. Typically, the exponential distribution is used to model times between events, but here we used it to model the fluctuations in the values of the counts across the genes, as they randomly fluctuate their expression across each of the 54 tissues reported in the GTEx portal. We note this to underscore that the results spontaneously self-emerge from the random combination of the genes involved (with and without removal), rather than from the clinical criteria used to denote gene relevance to autism, or the evidence from the literature used to determine their association. There is in fact no scoring of 
such relevance for the genes associated with the other neurological disorders under consideration (e.g., ataxias and Parkinson's disease) or for the autistic disorders reported in the DisGenNet portal. Using those genes instead of the SFARI genes reveals tissues in Appendix B Table A4, where we report the convergence.

A

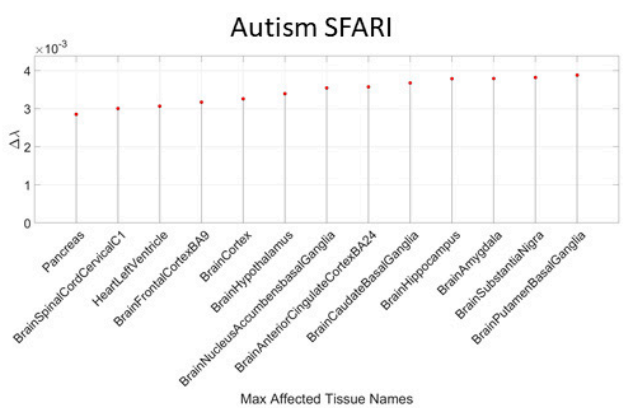

C

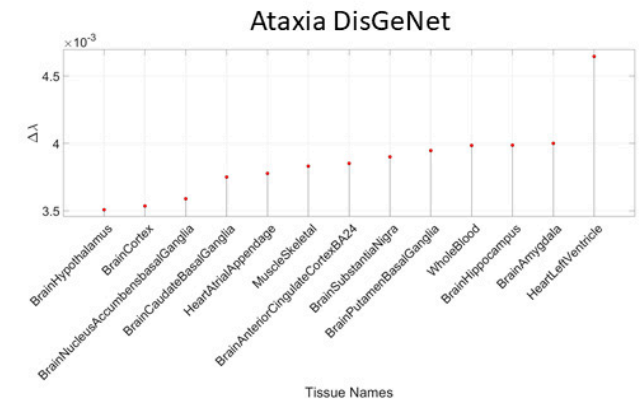

B

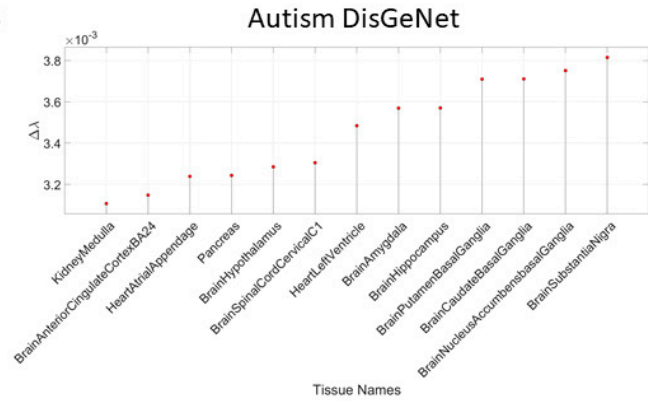

D

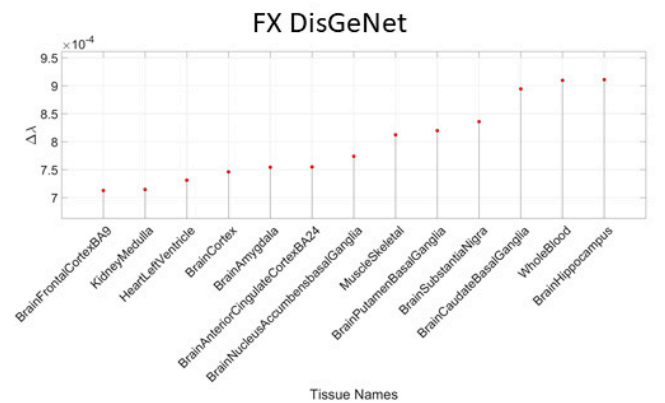

Figure 4. Convergence between autism and known neurological disorders shown by comparison of maximally affected tissues after removing genes associated with the disorder in autism (from the SFARI), autistic disorders (A,B) (from the DisGeNet portal) vs. ataxia (C) and fragile X (D) (See Appendix B Tables A3 and A4).

Removal of the SFARI genes, ranked by change in gene expression, reveals brain tissues linked to the CNS, in brain subcortical tissues linked to motor control (basal ganglia, striatum), memory (hypocampus), emotions (amygdala) and regulation (hypothalamus); and the spinal cord. This is also generally the case for the removal of the DisGeNet genes associated with autistic disorders and with FX and ataxia. Congruent with the outcome from the removal of the SFARI genes from the GTEx genome, the DisGeNet gene removal also affected the tissues associated with CNS function. Important tissues for systemic organ functioning such as those containing smooth muscles, cardiac and skeletal muscles in the taxonomy proposed in Figure 1B were also affected (commonly) across these disorders. Figure 4 shows a summary of the results, visualizing the stochastic shifts. Appendix B Tables A3 and A4 show the tissues ranked in descending order and color coded according to CNS (brain and spinal cord in blue), heart related (pink), muscle-skeletal (green), and peripheral vital organs (gray). Most tissues in autism and the neurological disorders are from the CNS, followed by PNS-related tissues in the heart and muscle-skeletal and with vital organs towards the end of the $\Delta \lambda$ ranking.

Supplementary Figures S1-S3 show these results separately for each neurological condition. We note in Supplementary Materials that removal of the SFARI autism syndromic genes from the GTEx genome reveals maximal differences in tissues of organs with smooth and cardiac muscles linked to involuntary and autonomic function in the proposed taxonomy of Figure 1B.

Removal of the overlapping SFARI genes and neurological disorders also reveal brain tissues linked to motor control, memory, emotions, and regulation. This is depicted in Figure 4. Given the congruence between the tissues maximally affected by removing the SFARI autism genes from the GTEx database and those from the neurological conditions, we next ascertain the extent to which these 
genes overlap with those used from ataxias in the literature. To that end, we divide them into the autosomal dominant, the autosomal recessive and the X-chromosome genes. Figure 5A shows the result upon removal of overlapping genes between the SFARI autism set and ataxias (dominant and recessive and X-chromosome sets) from the literature. Appendix B Table A3, Column 3 lists the tissues, while Appendix B Table A5 also has the scoring from the SFARI autism genes. Supplementary Materials Table S2 lists the phenotypic information of the disorders associated with these genes, as described by the clinical literature. Figure 5B lists the PD gene that overlaps with the SFARI autism genes, also depicted in Appendix B Table A5 along with the score ranking from the SFARI portal.
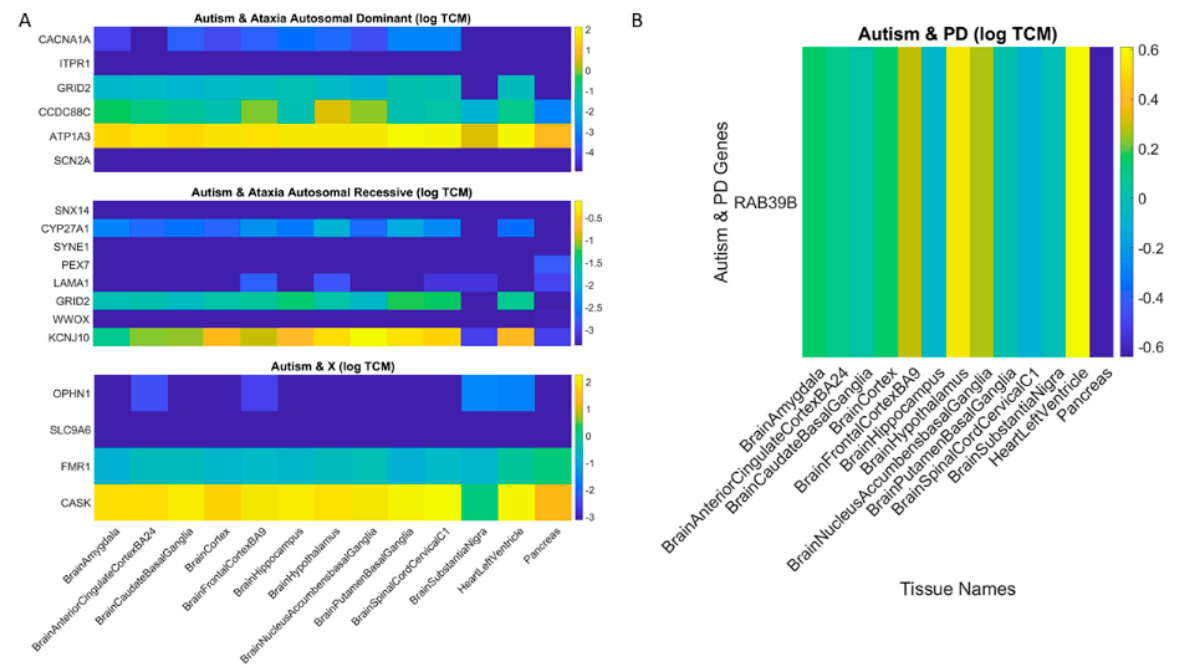

Figure 5. Gene expression on maximally affected tissues (color bar coded in log TPM) upon removal of overlapping genes between the SFARI autism set and ataxias (dominant and recessive and X-chromosome sets) from the literature (A) and (B) from Parkinson's disease. The horizontal axis lists the tissue names and the vertical axis lists the gene names.

We note that removing this subset of 14 overlapping genes from the SFARI autism set (Appendix B Table A5) does not change the primary result, whereby the most-affected tissues upon removal of the SFARI autism set from the GTEx dataset are those associated with subcortical brain structures critical for motor control, adaptation/learning, regulation, coordination and autonomic function as well as memory and emotion. This is shown in Figure 5A,B and in the third column of Appendix B Table A3. In Figure 4D, we also plot the top-ranked tissues affected by the removal of the FX genes reported in the DisGeNet portal from the GTEx dataset. Supplementary Figures S19-S22 further provide details on X-chromosome genes in Figure 5A implicated in autism according to the SFARI genes portal.

The result that convergence in the ranked descending order of the CNS (brain and spinal cord tissues), followed by heart-related tissues, muscle-skeletal tissue and lastly peripheral vital organs for systemic functioning in the SFARI autism and well-known neurological disorders from the literature is also congruent with the results using the genes associated with these conditions in the DisGeNet portal. There, we interrogated autistic disorders, ataxias and fragile $X$, confirming the overlap in genes, their expression on the 54 tissues of the GTEx database and the orderly levels of tissues maximally affected by the removal of the associated genes. We grouped the tissues by CNS, heart, muscle-skeletal and peripheral vital organs to follow the proposed taxonomy of Figure 1B.

In the remaining sections of this paper, we consistently use this tissue grouping to simplify visualization of the Appendix B tables and data presentation. Figure 6 shows the results for different types of ataxias and FX, while Appendix B Table A6 summarizes the top-ranked affected tissues in different types of ataxias. These are color coded according to the tissue grouping, approximating the taxonomy proposed in Figure 1B. 
A

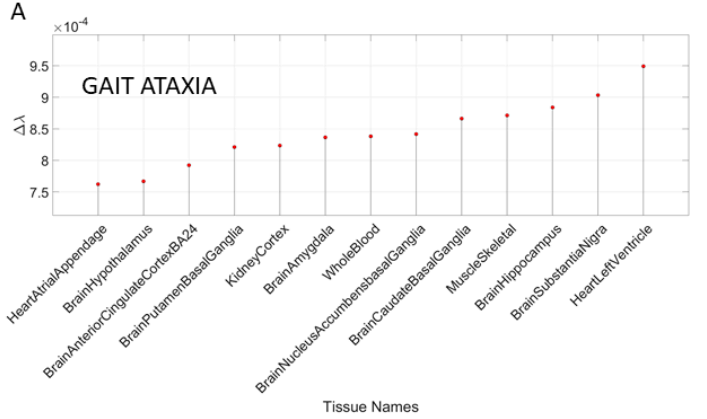

$\mathrm{C}$

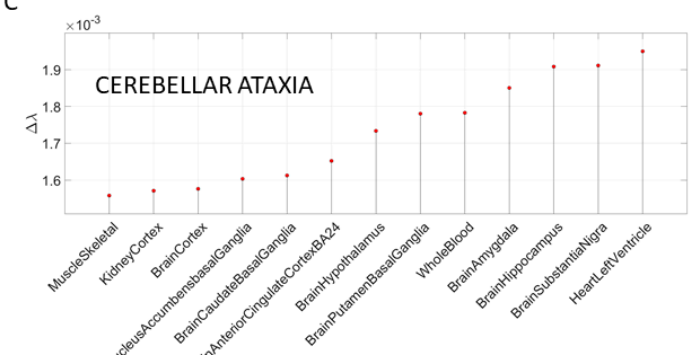

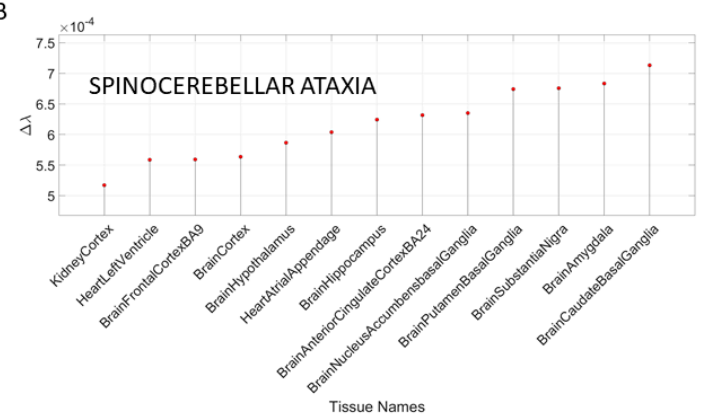

D

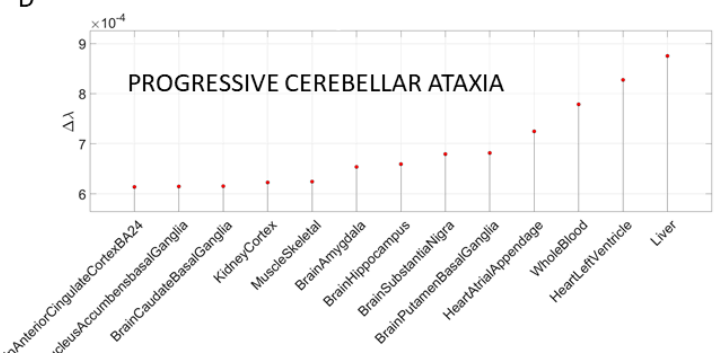

Figure 6. Different types of ataxia showing the top $\Delta \lambda$-ranked tissues, also shown in Appendix B Table A7.

(A) Gait ataxia; (B) spinocerebellar ataxia; (C) cerebellar ataxia; (D) progressive cerebellar ataxia.

3.2. Removal of Genes Associated with Schizophrenia and Multiple Forms of Mental Depression Reveals Convergence with Neurological Disorders

The removal of the DisGeNet genes associated with mental illnesses such as schizophrenia, depression, bipolar depression and unipolar depression from the normative GTEx genome resulted in convergence of maximally affected tissues involved in the CNS, especially those brain regions necessary for neuromotor control, memory, and emotion. This is depicted in Appendix B Table A7 and Figure 7. Several of these tissues were also found to be affected upon removal of the SFARI genes and the genes associated in DisGeNet with autistic disorders. Furthermore, these are maximally affected tissues in the well-known neurological conditions depicted in Figures 4 and 5 and Appendix B Tables A3-A5.
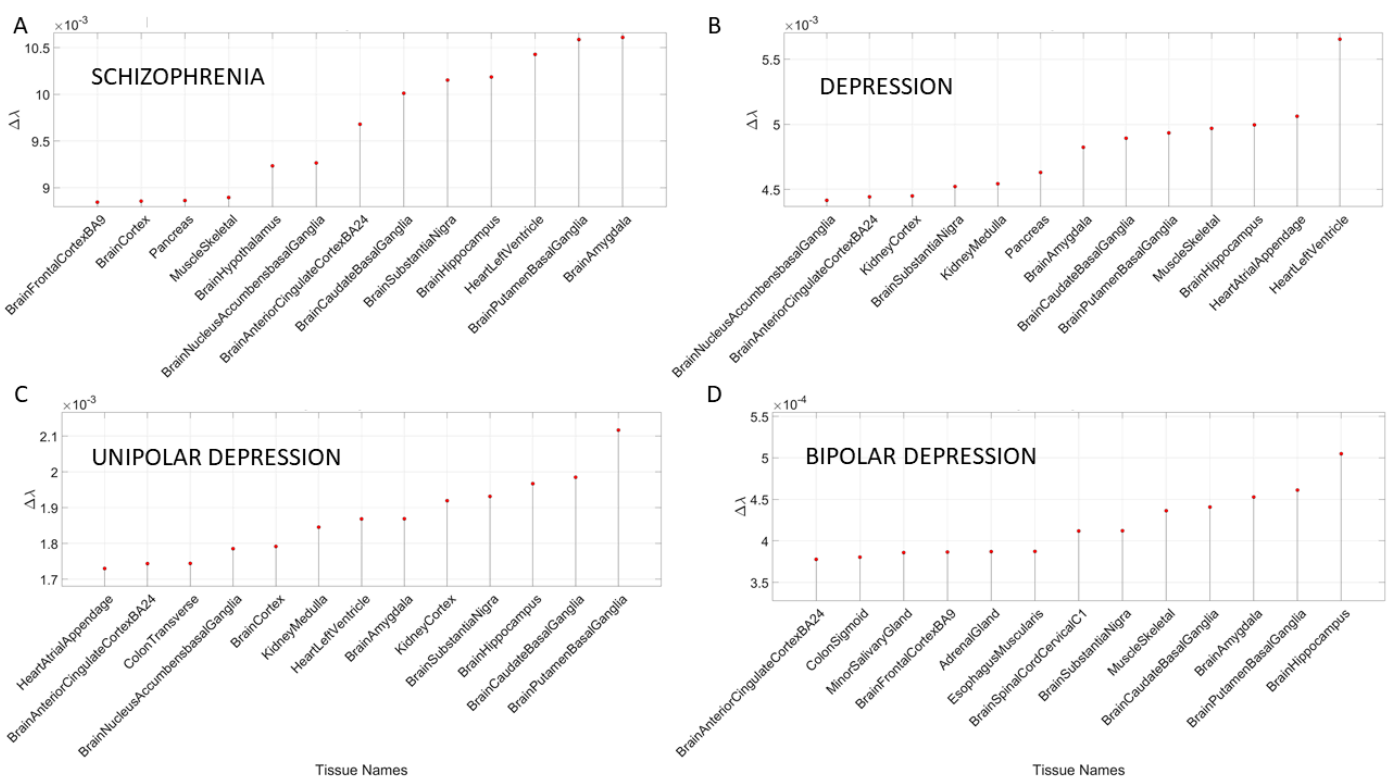

Figure 7. Maximally affected tissues in schizophrenia (A) and depression (B), and in different types of depression (unipolar (C) and bipolar (D)) shown in Appendix B Table A7. 


\subsection{Removal of Genes Associated with Non-Neurological Disorders Reveals Other Non-CNS Tissues}

In addition to the examination of mental illnesses and neurological disorders, we also interrogated the GTEx genome upon removal of genes associated with various non-neurological disorders. These included various forms of cancers, inflammatory and autoimmune disorders and other tissues related to the heart, the circulatory and the endocrine systems. Appendix B Tables A8 and A9 summarize the results of this interrogation and Figures 8 and 9 show the $\Delta \lambda$-ranking graphs.
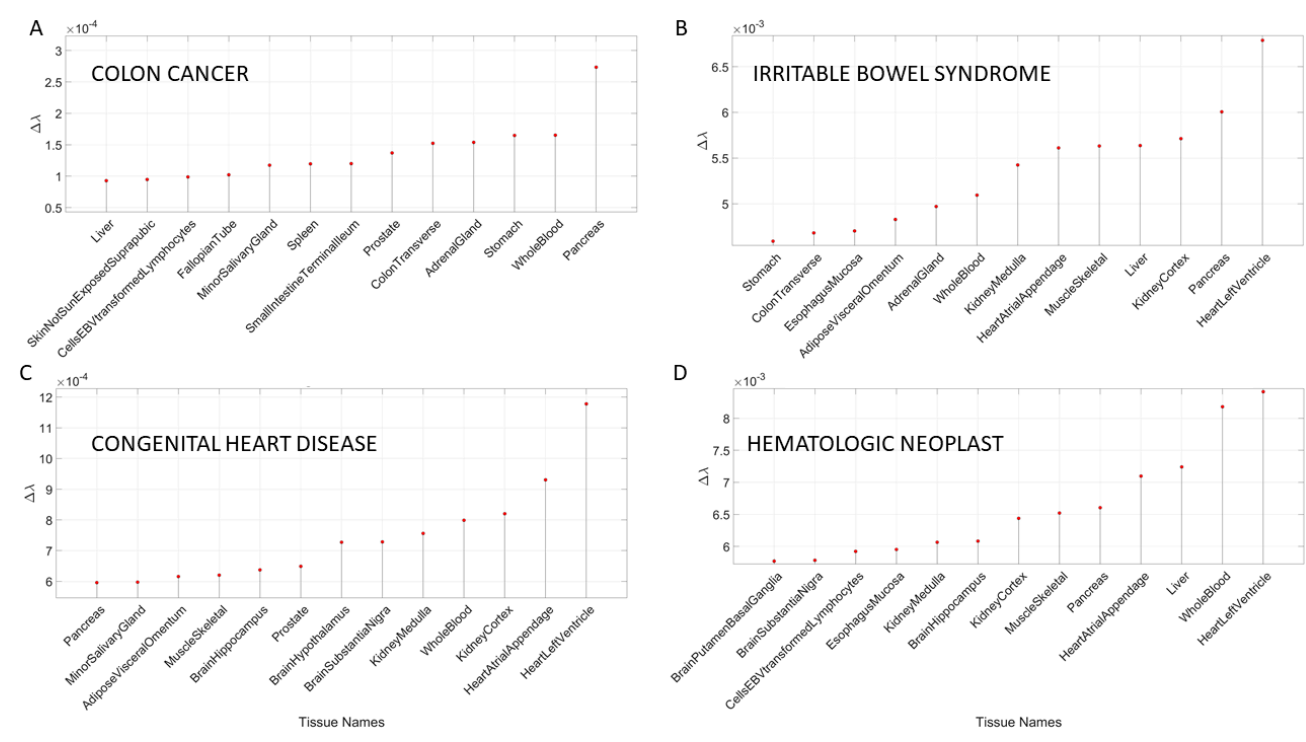

Figure 8. Maximally affected tissues in a sample non-neurological diseases of Appendix B Table A8 reveal primarily non-CNS tissues involving peripheral vital organs for systemic functioning, followed by heart-related and muscle-skeletal tissues. As before, the interrogation of the GTEx genome is based on the genes associated with diseases in the DisGeNet portal. (A) Colon cancer; (B) irritable bowel syndrome; (C) congenital heart disease and (D) hematologic neoplast. Color code as in previous tables.

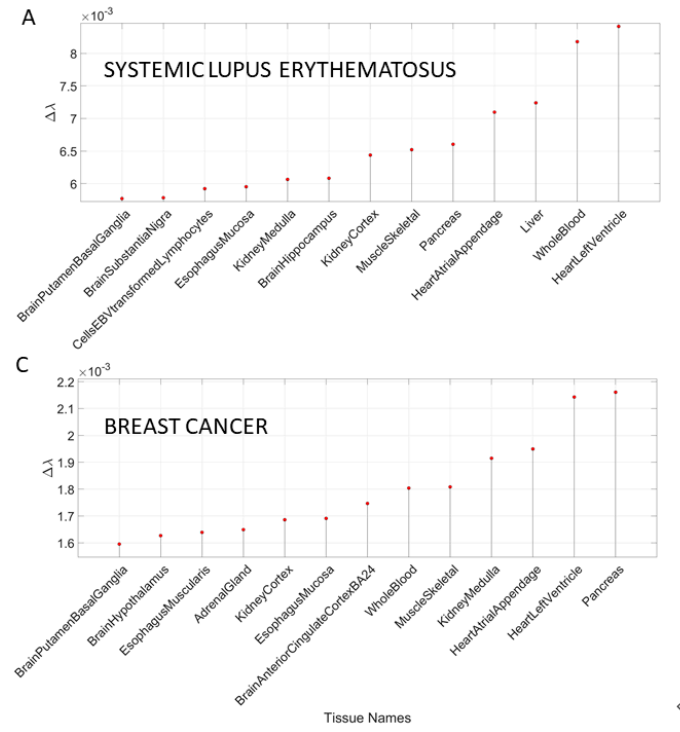

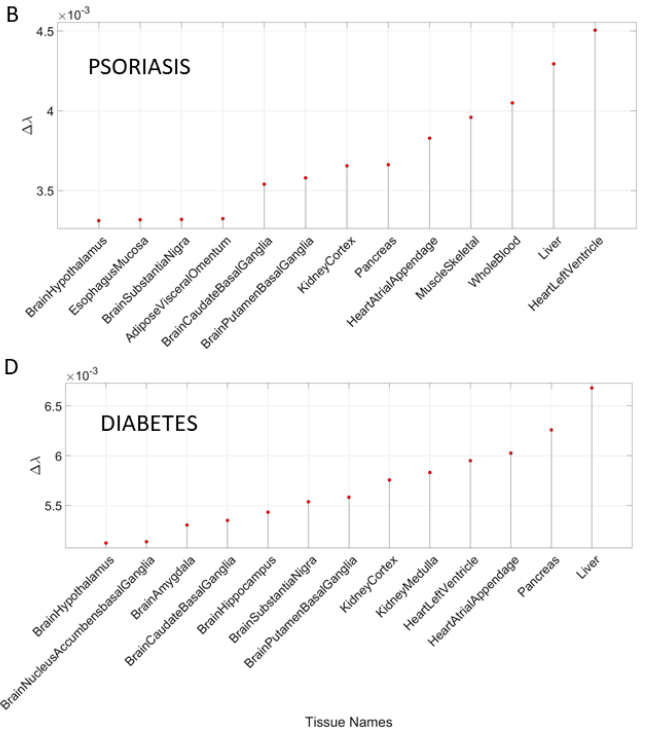

Figure 9. Maximally affected tissues in non-neurological diseases. (A) Systemic Lupus Erythematosus; (B) Psoriasis; (C) Breast Cancer; (D) Diabetes. These are depicted in Appendix B Table A10 median-ranked according to the $\Delta \lambda$ values obtained from the absolute difference between the tissues according to the full genome in the GTEx database and the GTEx genome without the genes associated with each disease, according to the queries to the DisGeNet portal. 
The results of the maximally affected tissues upon the removal of the genes associated with these non-neurological disorders revealed a very different picture than those upon removal of the genes associated with the mental illnesses (autism, schizophrenia and the depressions) and those associated with the known neurological conditions (the various forms of ataxia, FX and Parkinson's disease). Namely, the CNS-related tissues were less affected in these non-neurological diseases than those related to the PNS (muscle-skeletal and ANS heart), and those linked to peripheral bodily organs were the most visibly affected. The exception was diabetes, maximally affected tissues in peripheral organs, but also CNS and PNS tissues in the tail of the top $\Delta \lambda$-ranked tissues. We next interrogate the genome in relation to mitochondrial disorders of several kinds and acquired PTSD.

\subsection{Removal of Genes Associated with Mitochondrial Diseases Reveals that Heart-Related Tissues Are Maximally Affected but PTSD Is Mixed}

Removal of genes associated with mitochondrial disorders of various types from the GTEx genome, according to the genes in the DisGeNet portal, reveal a mixture of tissues associated with peripheral vital organs for systemic functions, heart-related and muscle-skeletal- and CNS-related tissues. The top half of the highest-ranked tissues in mitochondrial disease shows affected tissues related to the heart, muscle-skeletal and peripheral organs, while the bottom half shows more involvement of brain-related tissues in subcortical regions of motor control. In contrast, mitochondrial myopathies show a predominance of CNS-related tissues, including the brain and spinal cord, with top $\Delta \lambda$-ranked tissues related to the heart and muscle-skeletal tissues. Mitochondrial encephalopathy, lactic acidosis, and stroke-like episodes (MELAS) show a predominance of tissues associated with peripheral vital organs for systemic function and heart-related tissues. Only two brain regions for motor control and emotion are present in the bottom-ranked tissues of the most-affected tissues.

The case of acquired PTSD also reveals a mixture of tissues from brain, heart, and peripheral organs. There, we see maximally affected tissues linked to subcortical regions of the brain involved in motor control, adaptation, learning, and coordination intermixed with tissues linked to peripheral bodily organs (like the kidneys) and the autonomic systems' heart. Furthermore, we also see tissues linked to the hypothalamus, a regulatory brain structure. Figure 10 shows the graphs of the $\Delta \lambda$ difference, which was median ranked, as in the previous cases, for these disorders.
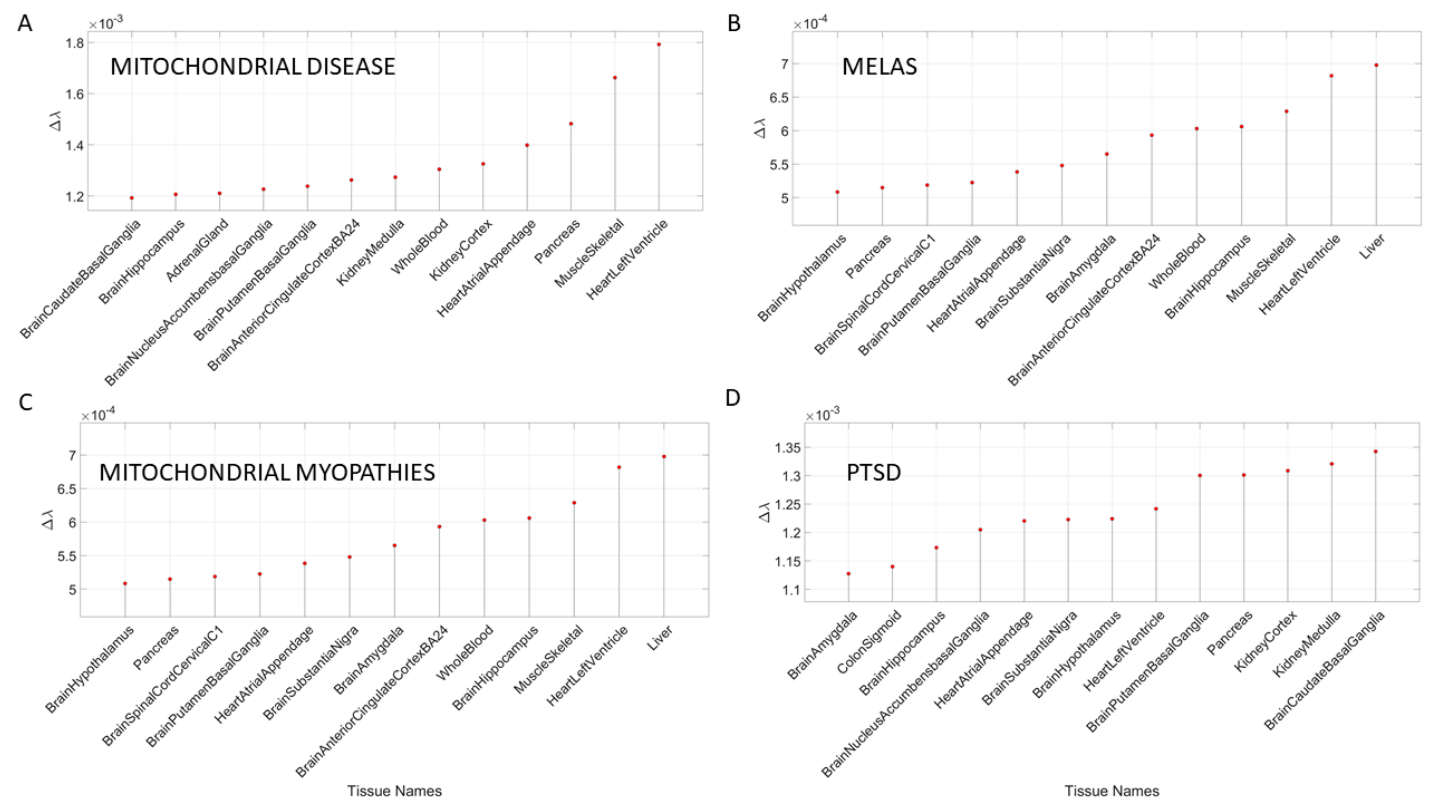

Figure 10. Maximally affected tissues in mitochondrial diseases (A-C) and in PTSD (D) of Appendix B Table A10, shown in graphical form according to the $\Delta \lambda$ values. 
We summarize the results across all 54 tissues (in alphabetical order from left to right) in Figure 11. Here, a color map depicts the values of $\Delta \lambda$ normalized for each disease (along the rows) across the tissues (columns) by dividing by the maximum $\Delta \lambda$ value of each row. The patterns reveal that the maximally affected tissues (upon genes removal) are common to both neurological disorders and mental illnesses. They correspond to the brain tissues involved in motor control, adaptation, and learning (basal ganglia, striatum, substantia nigra), tissues in involved in emotion (amygdala), memory (hippocampus) and systemic regulation (hypothalamus). They also reveal that whole blood tissue is not as affected in the mental illnesses as in the neurological disorders (marking a point of divergence that warrants further investigation). Heart-related tissues and muscle-skeletal tissues are also shared between these mental illnesses and neurological disorders when the genes specific to each disorder are removed from the GTEx genome. Interestingly, the pancreas is an example of a peripheral bodily organ with tissues that are commonly affected across most of the disorders and diseases interrogated here. Yet they have lower weight the neurological disorders compared to the non-neurological diseases under examination.

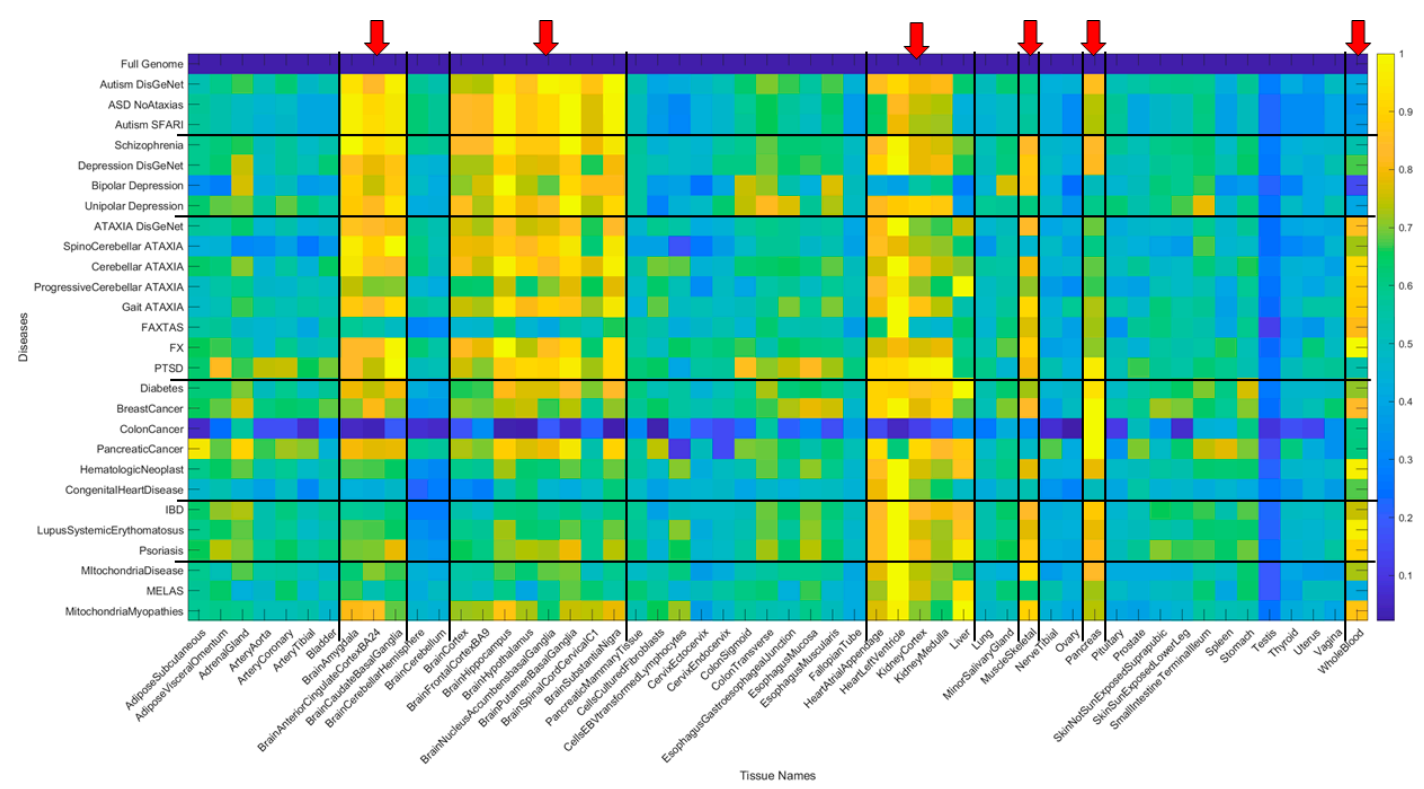

Figure 11. Summary of disorders/diseases (27 rows) $x$ tissues (54 columns) in alphabetical order. Entries are $\Delta \lambda$ (difference with respect to the gene expression values from the full GTEx genome) values normalized by the maximum across the tissues for each row (disorder/disease). The first row is the $0-\Delta \lambda$ difference reference from the full genome. Red arrows mark the maximally affected tissues across all diseases, showing mental illnesses on the top, followed by neurological disorders, then non-neurological, including several types of cancer, autoimmune disorders, and diabetes. Black lines delineate blocks of diseases (along rows) and blocks of gene expression on tissues (along columns).

The non-neurological diseases reveal less involvement of the CNS-related tissues but highly overlap with the heart and muscle-skeletal tissues. Tissues linked to the kidneys, liver and pancreas are also maximally affected by genes' removal in these diseases. Colon cancer shows an interesting pattern whereby the pancreas reveals maximal normalized $\Delta \lambda$ values. Figure 12 summarizes the patterns in binary form by turning ON (yellow) values above 0.8 considered high and OFF those below (blue). This cut off is chosen to further highlight overlap and differences across diseases based on high $\Delta \lambda$.

The patterns revealed by the high values of the normalized $\Delta \lambda$ quantity, show convergence of maximally affected brain tissues in mental illnesses with the neurological disorders but not with the non-neurological disorders (except for diabetes which does affect some brain regions.) The mitochondrial diseases do not show the same intensity of the CNS-related $\Delta \lambda$ values as the mental illnesses and the neurological disorders, but they do share the heart and muscle-skeletal patterns with 
all the examined diseases and disorders. This is interesting, given that some of the children with various forms of mitochondrial disorders may receive diagnoses of autism. In summary, there is clear overlap between mental illness and neurological disorders, suggesting involvement of the central nervous systems in both. We also see major contributions from the peripheral nervous systems, particularly the heart, the muscle-skeletal tissues and, to a lesser degree, tissues of peripheral organs. The latter are most affected in the non-neurological diseases. Figure 13 shows the most-affected tissue in each disease/disorder (also depicted in Appendix B Table A11).

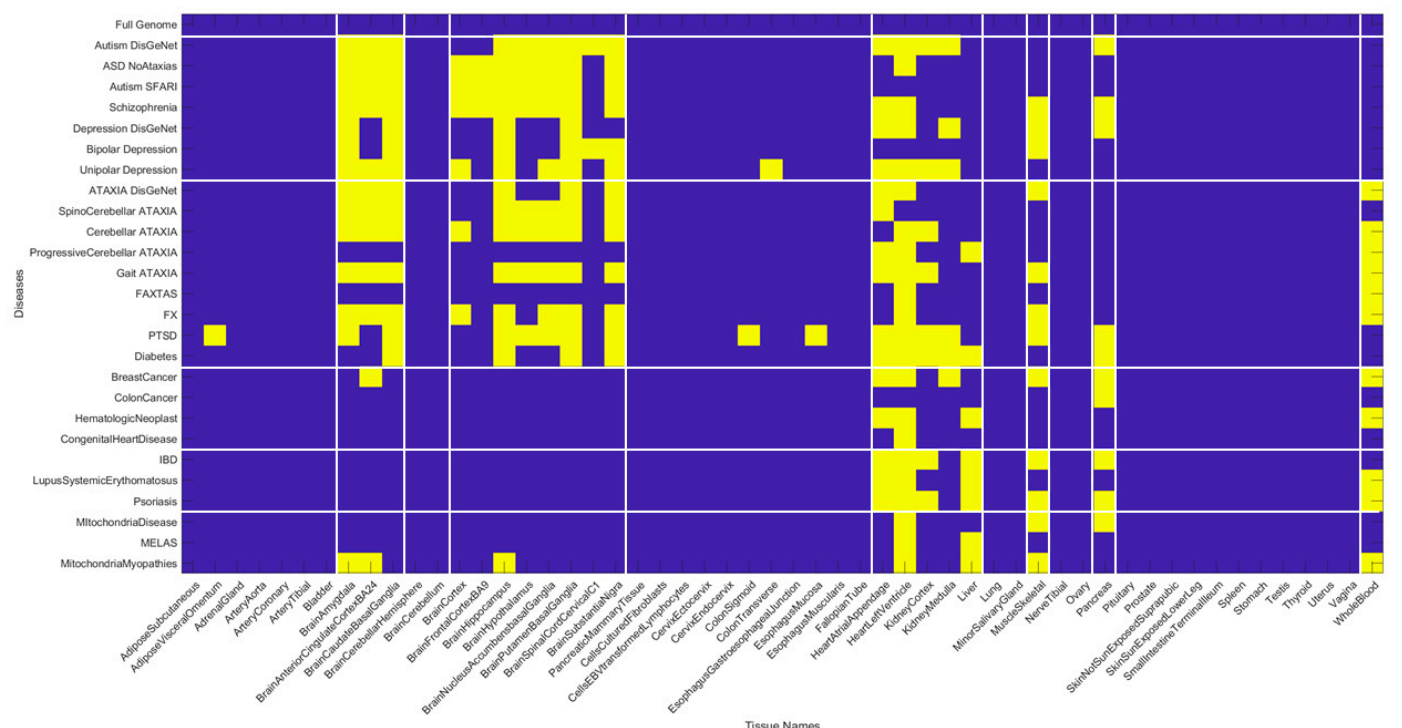

Figure 12. Binary version of the matrix in Figure 11 upon thresholding by a high normalized $\Delta \lambda$ value of 0.8 shows that the overlap across mental illnesses, neurological disorders and non-neurological diseases is primarily in the heart tissues, the muscle-skeletal tissues and organs such as the pancreas, liver, and kidney. Whole blood tissue is shared between neurological and non-neurological disorders but not present in the mental illnesses. Brain tissues are shared between mental illnesses and neurological disorders.
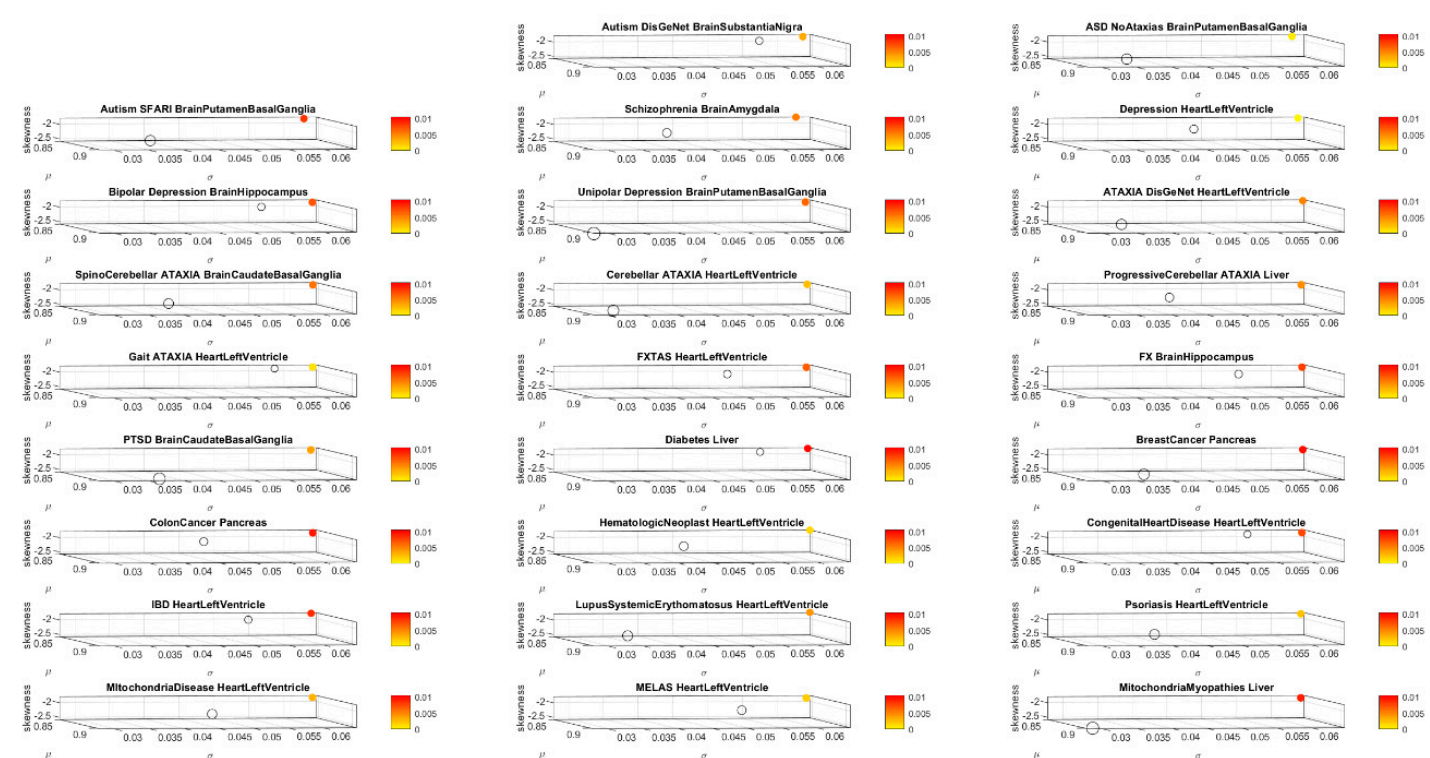

Figure 13. Stochastic shift of gene expression on tissue after gene removal for each disease/disorder under consideration (colored circle) relative to the gene expression on that tissue using the full genome 
in GTEx (open circles). The most-affected tissue in each disorder/disease under consideration was selected according to the maximum $\Delta \lambda$ value, the absolute difference between the empirically estimated $\lambda$ using MLE of the exponential distribution rate parameter for the full GTEx genome and for the genome minus the genes associated with each disease/disorder. Since the exponential distribution is a particular case of the continuous gamma family of probability distributions (when the gamma shape parameter is 1), we also used MLE to estimate the shape and scale gamma parameters and the four gamma moments, plotted here in a five-dimensional parameter space. Along the $x$ axis, we plot the empirically estimated gamma mean; along the $y$ axis, we plot the gamma variance; along the $z$ axis, we plot the gamma skewness and the kurtosis is used to represent the size of the marker (more kurtotic distributions have higher value, i.e., larger circle). The fifth dimension is the color representing the $\Delta \lambda$ (see also Figure 3C, visualizing one single disease and all 54 tissues or summarizing the top 13 median-ranked tissues as those most affected).

\section{Discussion}

This work interrogated the human genome by removing genes associated with various diseases and comparing the outcome from the remaining gene expression on 54 tissues commonly examined in the GTEx portal. These tissues involve parts of the central nervous systems (the brain and the spinal cord) and parts of the peripheral nervous systems (the muscle-skeletal tissue and the heart tissues as part of the autonomic nervous system), which we grouped in Figure 1B. Other tissues are from peripheral vital organs for systemic bodily functions (whole blood, pancreas, liver, kidneys, lungs, etc.).

We compared mental illnesses such as autism, schizophrenia, and various types of mental depression (including unipolar and bipolar), with well-known neurological disorders such as different types of ataxias, fragile $X$ and Parkinson's disease. We found convergence between the tissues maximally affected by the removal of disease-associated genes across these mental illnesses and neurological disorders. CNS-related tissues in subcortical regions of the brain related to motor control, motor learning, motor coordination, and motor adaptation, as well as memory and emotion, were predominantly maximally affected by the corresponding gene removal across both the mental illnesses and the neurological disorders. This convergence demonstrates overlap between psychiatric and neurological conditions with specific involvement of motor, memory, emotional and regulatory axes. In autism and FX, we obtained congruent results on maximally affected tissues. The results were consistent using removal of the genes from the SFARI autism database and using the genes upon querying the DisGeNet portal. In addition to the genes reported by querying DisGeNet, we also used the genes reported in the literature for schizophrenia and depression, and for Parkinson's disease. We found congruence in all cases.

To further test our hypothesis that these mental illnesses are disorders of the nervous systems and that removing the gene pool associated with them gives rise to overlapping tissues related to CNS functioning, we also queried DisGeNet about other non-neurological diseases. We found that in such cases, the predominance of maximally affected tissues was on tissues associated with peripheral vital bodily organs related to the disease, such as pancreas, kidney, liver, and colon transverse in colon cancer. Furthermore, several of these diseases had maximally affected heart-related tissues and whole blood. Other cases also revealed a predominance of peripheral organs. We lastly, interrogated the genome in relation to mitochondrial diseases and acquired PTSD. In these cases, we hypothesized and confirmed a mixture of tissues related to peripheral organs (for mitochondrial diseases) and the CNS (for PTSD).

In the case of mitochondrial diseases, the heart-related tissues were revealed as the most affected along with muscle-skeletal tissue. Furthermore CNS-related tissues were more affected by gene removal in mitochondrial myopathies (i.e., the amygdala and the anterior cingulate cortex), as compared to MELAS or mitochondrial disease. The common thread across all three types of mitochondrial-related disorders was the heart-related tissues. The case of acquired PTSD showed a mixture of CNS-related tissues, tissues related to bodily peripheral organs, and heart-related tissues. The kidney and pancreas were also affected in PTSD. When we examined the maximum $\Delta \lambda$ for each disorder/disease under 
examination, we found that the basal ganglia was maximally affected in autism, unipolar depression, spinocerebellar ataxia, and PTSD), while the heart left ventricle tissue was maximally affected in depression, ataxia, cerebellar ataxia, gait ataxia and fragile $X$ tremor ataxia syndrome (FXTAS). This result indicates overlap between the psychiatric mental illnesses and the neurological disorders. It also shows the importance of examining mental illnesses in a more systemic way that includes the autonomic nervous system of the PNS.

This test on non-neurological illnesses served as a control to show that removal of the genes associated with each disorder did have specificity with the disorder and yet a very different outcome when comparing the mental illnesses to the non-neurological disorders. Among the top tissues affected across non-neurological diseases, the pancreas was maximally affected by the removal of disease-associated genes in breast and colon cancer, while the liver was maximally affected in diabetes. The heart left ventricle was maximally affected across autoimmune disorders such as psoriasis, lupus systemic erythematosus, and irritable bowel disease (IBD). The heart was also maximally affected in hematologic neoplast, congenital heart disease, mitochondrial disease and MELAS, in contrast to the mitochondrial myopathies which showed the liver as the maximally affected tissue.

This exercise demonstrated that despite the stochastic nature of gene expression, upon removal and random recombination, there is convergence across psychiatric and neurological disorders, thus potentially rendering both as disorders of the nervous systems. In both cases, we found a strong prevalence of the CNS, but also found important differences in tissues from the PNS, including the heart and the muscle-skeletal tissues involved in both mental illnesses and neurological disorders. Because of these convergences, and the fact that there are treatments and accommodations to help persons with neurological disorders, it may be possible to leverage some of those types of bodily-based supports to help persons with mental illnesses. Behaviors that are described by observation to define mental illnesses can now be connected with underlying tissues involved in voluntary, involuntary, and autonomic function across the CNS and the PNS and mapped to the genome, thus closing the present gap between behaviors and genomics in the precision medicine knowledge network. In this sense, the present methods offer a new way to interrogate the genome and link tissues with behavioral phenotyping.

A surprising finding here is the potential contributions of peripheral structures and organs to mental illness. Tissues of the autonomic nervous systems were maximally impacted by the removal of the genes associated with these mental illnesses, as was the muscle-skeletal tissue among the top-ranked illnesses. Tissues associated with subcortical brain regions necessary for motor control, learning, adaptation, and coordination (basal ganglia and striatum) were highly impacted by the removal of the genes in both mental illnesses and neurological disorders, along with those tissues important for memory (hippocampus), emotion (amygdala) and regulation (hypothalamus). Surprisingly, we did not see cerebellum-related tissues among the most affected by the removal of the genes (even in ataxias) where we do know that the cerebellum plays a large role [20-23]. This was also the case in autism, where we know the cerebellum has been implicated [24,25].

Lastly, the autoimmune disorders that we examined had very different brain tissue patterns from the mental illnesses and neurological conditions but shared the heart-related tissues and the muscle-skeletal tissue. In this sense, the contributions from the peripheral systems to mental illnesses and to autoimmune disorders seem important. However, blood tissue marked a departure of neurological disorders from mental illnesses, as it was maximally affected in neurological disorders but not in the mental illnesses. Overall, these gene removals revealed surprising results that invite rethinking how we may want to describe, diagnose, and treat mental illnesses in general.

\section{Caveats and Future Directions}

Although we found evidence that the mental illnesses and neurological disorders have remarkable overlap in the types of brain tissues that are maximally affected by the removal of their corresponding associated genes, we recognize that gene removal is a crude way to interrogate the human genome 
and its expression of the 54 tissues of the GTEx database. Future work will aim at developing more sophisticated methods to explore gene overexpression and to build simulations of the use of these methods in, e.g., combination with dynamic transcriptome evolution during neuronal differentiation in the development of cell lines from induced pluripotent stem cells. This will be important to move beyond a static approach and be able to assess asynchronous gene expression behaviors over time when cell lines differentiate into neuronal types. Full transcriptome dynamic interrogation longitudinally, over time, is now possible using these stochastic analyses in combination with the various data repositories featuring disease-associated genes.

The present work merely scratches the surface on possible new ways to interrogate the human genome in relation to diseases of all types (not just mental or neurological) in order to possibly build comparative models of outcomes in tissues that can be related to behavioral phenotypic manifestations of the clinical disorder. In this sense, the work presented here can help bridge the gap between behavioral description of a mental illness, or a neurological disorder, and its genetic underpinnings via the affected tissues. Combining this approach with the new wave of digital biomarkers that describe human behavior digitally at a microscopic level [9,26-29], using objective means and a finer level of granularity beyond naked eye detection, could help us redefine many psychiatric disorders and medical conditions under the precision medicine paradigm.

\section{Conclusions}

We here offer a new roadmap to reframe psychiatry using the precision medicine paradigm. The new stochastic approach can initiate the steps to connect behavioral phenotypic description from clinical observation and digital characterizations therein, with the underlying neurobiology of mental illnesses. Borrowing knowledge from neurology and brain science, it will be possible to shift psychiatry from an art to a quantitative objective science under the tenets of precision medicine by integrating all layers of the knowledge network. This would help design personalized targeted treatments utilizing the person's genome, localizing the most-affected tissues defining central nervous system functions and distinguishing those from tissues related to vital organs for systemic functions. This new approach could potentially mark the beginning of a transformative era in mental health.

\section{Patents}

E.B.T. holds the US Patent "Methods and Systems for the Diagnoses and Treatments of Nervous Systems Disorders" combined in this paper as micro-movement spikes (MMS) data type and continuous gamma probability distribution family empirical estimation.

Supplementary Materials: The following are available online at http://www.mdpi.com/2075-4426/10/4/144/s1, Figure S1: Outcome of genes' expression on most affected tissues upon removing the Ataxia genes from the normative data., Figure S2: Outcome of genes' expression on most affected tissues upon removing the FX and PD genes from the normative data., Figure S3: Outcome of genes' expression on most affected tissues upon removing the Mitochondrial disease genes from the normative data, Figure S4: Outcome of genes' expression on most affected tissues upon removing genes based on SFARI Autism scores., Figure S5: Information on gene CACNA1C common to the SFARI Autism set and the Ataxia Autosomal Dominant genes reported in the literature., Figure S6: Information on gene SCN2A common to the SFARI Autism set and the Ataxia Autosomal Dominant genes reported in the literature, Figure S7: Information on gene ATP1A3 common to the SFARI Autism set and the Ataxia Autosomal Dominant genes reported in the literature., Figure S8: Information on gene CCDC88C common to the SFARI Autism set and the Ataxia Autosomal Dominant genes reported in the literature., Figure S9: Information on gene ITPR1 common to the SFARI Autism set and the Ataxia Autosomal Dominant genes reported in the literature., Figure S10: Information on gene KCNJ10 common to the SFARI Autism set and the Ataxia Autosomal Recessive genes reported in the literature., Figure S11: Information on gene WWOX common to the SFARI Autism set and the Ataxia Autosomal Recessive genes reported in the literature., Figure S12: Information on gene GRID2 common to the SFARI Autism set and the Ataxia Autosomal Recessive genes reported in the literature., Figure S13: Information on gene LAMA1 common to the SFARI Autism set and the Ataxia Autosomal Recessive genes reported in the literature., Figure S14: Information on gene PEX7 common to the SFARI Autism set and the Ataxia Autosomal Recessive genes reported in the literature., Figure S15: Information on gene SYNE1 common to the SFARI Autism set and the Ataxia Autosomal Recessive genes reported in the literature., Figure S16: Information on gene CYP27A1 common to the SFARI Autism set and the Ataxia Autosomal Recessive genes reported in the literature., Figure S17: Information on gene SNX14 common to the SFARI Autism set and the Ataxia 
Autosomal Recessive genes reported in the literature., Figure S18: Information on gene RAB39B common to the SFARI Autism set and the Ataxia Autosomal Recessive genes reported in the literature., Figure S19: Information on gene CASK common to the SFARI Autism set and the X-Chromosome genes reported in the literature., Figure S20: Information on gene FMR1 common to the SFARI Autism set and the X-Chromosome genes reported in the literature., Figure S21: Information on gene SLC9A6 common to the SFARI Autism set and the X-Chromosome genes reported in the literature., Figure S22: Information on gene OPHN1 common to the SFARI Autism set and the X-Chromosome genes reported in the literature., Table S1: Genes common to the SFARI Autism set and the Ataxias and PD-early onset literature.

Funding: This research was funded by the New Jersey Governor's Council for Medical Research and Treatments of Autism and by the generosity of the Nancy Lurie Marks Family Foundation.

Acknowledgments: I thank the SFARI, GTex and DisGeNet researchers for the curation and maintenance of their genes module and the compilation of the literature database supporting these repositories.

Conflicts of Interest: The author declares no conflict of interest. The funders had no role in the design of the study; in the collection, analyses, or interpretation of data; in the writing of the manuscript, or in the decision to publish the results.

\section{Appendix A}

TPM explanation from the site: "Transcripts Per Million (TPM) is a normalization method for RNA-seq, should be read as for every 1,000,000 RNA molecules in the RNA-seq sample, $x$ came from this gene/transcript. For each transcript in the gene model, the number (raw count) of reads mapped is divided by the transcript's length, giving a normalized transcript-level expression. The distribution of ambiguous reads (between transcripts of the same gene, or between different genes) is handled by OmicSoft's RSEM implementation. The sum of ALL normalized transcript expression values is divided by 1,000,000, to create a scaling factor. Each transcript's normalized expression is divided by the scaling factor, which results in the TPM value. Gene-level TPM's are calculated by summing up the transcript-level TPM for each gene. In this scaling, the sum of all TPMs (transcript-level or gene-level) should always equal 1,000,000. For cells that have approximately the same number of transcripts-per-cell, the TPM expression values can be compared between these cells to estimate relative abundance. For a given sample, TPM values will linearly scale with FPKM values for genes or transcripts, but FPKM will not add up to 1,000,000, so TPM can also be thought as FPKM, scaled to sum to $1,000,000^{\prime \prime}$.

Derivation of Maximum Likelihood Estimation of the rate parameter in the Exponential Distribution:

We estimate the likelihood $L\left(\lambda \mid x_{1}, x_{2}, \ldots, x_{n}\right)$, where $x_{i}$ is the series of counts representing the gene expression on each given tissue and $i$ ranges from 1 to $n$, the number of genes.

$$
\begin{aligned}
L\left(\lambda \mid x_{1}, x_{2}, \ldots, x_{n}\right) & =\lambda e^{-\lambda x_{1}} \lambda e^{-\lambda x_{2}} \ldots \lambda e^{-\lambda x_{n}} \\
& =\lambda^{n}\left(e^{-\lambda x_{1}} e^{-\lambda x_{2}} \ldots e^{-\lambda x_{n}}\right) \\
& =\lambda^{n}\left(e^{-\lambda\left(x_{1}+x_{2}+\ldots+x_{n}\right)}\right)
\end{aligned}
$$

To obtain the maximum likelihood, we take the derivative of the likelihood in Equation (A1) and set it to 0 (since the derivative is 0 at the maximum likelihood value).

$$
\frac{d}{d \lambda} L\left(\lambda \mid x_{1}, x_{2}, \ldots, x_{n}\right)=\frac{d}{d \lambda} \lambda^{n}\left(e^{-\lambda\left(x_{1}+x_{2}+\ldots+x_{n}\right)}\right)
$$

We take the log here because the derivative of the function and the derivative of the log of the function equals 0 at the same point. So, for the purposes of finding where the derivative is 0 , the original function in Equation (A2) and the log of it are interchangeable.

$$
\begin{aligned}
& \frac{d}{d \lambda} \log \left(\lambda^{n}\left(e^{-\lambda\left(x_{1}+x_{2}+\ldots+x_{n}\right)}\right)\right)=\frac{d}{d \lambda} \log \lambda^{n}+\log \left(e^{-\lambda\left(x_{1}+x_{2}+\ldots+x_{n}\right)}\right) \\
& \frac{d}{d \lambda} n \log \lambda-\lambda\left(x_{1}+x_{2}+\ldots+x_{n}\right)=0 \\
& n \frac{1}{\lambda}-\left(x_{1}+x_{2}+\ldots+x_{n}\right)=0 \\
& \lambda=\frac{n}{\left(x_{1}+x_{2}+\ldots+x_{n}\right)}
\end{aligned}
$$


Further, with this result in Equation (A3), we can obtain the maximum likelihood estimate of each $\lambda_{j}$, given all the 56,146 genes expressed with some random value for each of the $j=1: 54$ tissues.

\section{Appendix B}

Table A1. Gene distributions used in the removal process and literature sources.

\begin{tabular}{ccc}
\hline Neurological & Number of Genes & Source \\
\hline Parkinson's disease & 17 & Lit Review [14,16-19] \\
Ataxia Autosomal Recessive & 70 & Lit Review [11,12] \\
Ataxia Autosomal Dominant & 46 & Lit Review [11,12] \\
X-chromosome & 6 & Lit Review [11,12] \\
Fragile X (SFARI scores) & $73^{1}(17,11,30,15)$ & SFARI Genes Module \\
Fragile X Syndrome & 194 & DisGeNet \\
FXTAS & 62 & DisGeNet \\
Ataxia & 813 & DisGeNet \\
cerebellar Ataxia & 421 & DisGeNet \\
gait Ataxia & 159 & DisGeNet \\
Progressive cerebellar Ataxia & 134 & DisGeNet \\
Spinocerebellar Ataxia & 145 & DisGeNet \\
\hline Neuropsychiatric (DSM) & Number of Genes & Source \\
\hline autism (SFARI scores) & $906^{1}(144,216,468,78)$ & SFARI Genes Module \\
Autistic Disorder & 1043 & DisGeNet \\
schizophrenia & 2697 & Lit Review [30-35] and DisGeNet \\
Mental depression & 1468 & DisGeNet \\
depression Unipolar & 641 & DisGeNet \\
depression Bipolar & 116 & DisGeNet \\
\hline
\end{tabular}

${ }^{1}$ SFARI scores for autism and FX genes were also used to assess each scored module separately. FXTAS stands for fragile $\mathrm{X}$ tremor ataxia syndrome.

Table A2. Genes associated with non-neurological diseases.

\begin{tabular}{ccc}
\hline Non-Neurological & Number of Genes & Source \\
\hline Colon Cancer & 3669 & DisGeNet \\
Diabetes Mellitus (non-insulin & 3134 & DisGeNet \\
dependent) & & \\
Estrogen Receptor-Positive Breast & 510 & DisGeNet \\
Cancer & 267 & DisGeNet \\
Congenital Heart Disease & 827 & DisGeNet \\
Hematologic Neoplasm & 1883 & DisGeNet \\
Systemic Lupus Erythematosus & 1308 & DisGeNet \\
Psoriasis & 1483 & DisGeNet \\
Irritable Bowel Syndrome & Number of Genes & Source \\
\hline Mixed & 41 & Lit Review [13] \\
Mitochondria & 121 & DisGeNet \\
Mitochondrial Myopathies & 284 & DisGeNet \\
Mitochondrial Diseases & 81 & DisGeNet \\
MELAS Syndrome & 418 & DisGeNet \\
PTSD &
\end{tabular}

MELAS (a form of dementia) stands for mitochondrial encephalopathy, lactic acidosis, and stroke-like episodes.

The data file name from the GTEx portal https://www.GTExportal.org/home/datasets used in this paper is GTEx_Analysis_2017-06-05_v8_RNASeQCv1.1.9_gene_median_tpm.gct.csv (accessed on 18 September 2020).

The data file name from the SFARI genes is located at https:/gene.sfari.org/database/humangene/ and named SFARI-Gene_genes_03-04-2020release_03-05-2020export.csv (accessed on 18 September 2020). 
Table A3. The 13 top median-ranked tissues in descending order of $\Delta \lambda$ value, the most-affected tissues upon removal of the SFARI genes (906) linked to autism from the human GTEx database in column 1. Ataxias, $X$-chromosome, fragile $X$, Parkinson's disease and mitochondrial disease extracted from the literature and column 3 is the same as in column 1 while removing from the SFARI autism set 14 genes that overlap with ataxias and PD (see those genes listed in Supplementary Table S2). Tissues are grouped by CNS (brain and spinal cord in blue); muscle-skeletal (green), heart (pink) and peripheral organs (gray). SFARI autism (11/13 (84.6\%) CNS, 1/13 (7.6\%) heart and 1/13 (7.6\%) peripheral organ); neurological disorders (10/13 (76.9\%) CNS, 2/13 heart (15.3\%) and 1/13 (7.6\%) muscle-skeletal); SFARI autism without the overlapping genes from the neurological disorders (11/13 (76.9\%) CNS, 1/13 (7.6\%) heart and 1/13 (7.6\%) peripheral organs).

\begin{tabular}{ccc}
\hline SFARI Autism & Ataxias, $\mathbf{X}, \mathbf{F X}, \mathbf{P D}$, Mitochondria & $\begin{array}{c}\text { SFARI Autism without } \\
\text { Overlapping Ataxia, PD Genes }\end{array}$ \\
\hline Putamen Basal Ganglia & Hippocampus & Putamen Basal Ganglia \\
Substantia Nigra & Amygdala & Substantia Nigra \\
Amygdala & Aubstantia Nigra & Amygdala \\
Hippocampus & Heart Left Ventricle & Hippocampus \\
Caudate Basal Ganglia & Whole Blood & Caudate Basal Ganglia \\
Anterior Cingulate Cortex & Muscle Skeletal & Nucleus Accumbent Basal Ganglia \\
Nucleus Accumbent Basal Ganglia & Nucleus Accumbent Basal Ganglia & Anterior Cingulate Cortex \\
Hypothalamus & Putamen Basal Ganglia & Hypothalamus \\
Brain Cortex & Anterior Cingulate Cortex & Brain Cortex \\
Frontal Cortex & Brain Cortex & Frontal Cortex \\
Heart Left Ventricle & Hypothalamus & Spinal Cord \\
Spinal Cord & Heart Atrial Appendage & Heart Left Ventricle \\
Pancreas & Caudate Basal Ganglia & Kidney Cortex \\
\hline
\end{tabular}

Table A4. Most-affected tissues upon removal of the DisGeNet genes associated with autistic disorders (1043) from the human GTEx database (column 1); ataxia (813 genes) in DisGeNet (column 2) and FX (194 genes) in DisGeNet (column 3). Convergence between autism and neurological disorders is noted in the shaded tissues color coded as in Table A4, based on CNS, heart, and peripheral organs.

\begin{tabular}{ccc}
\hline DisGeNet Autistic Disorders & DisGeNet Ataxia & FX DisGeNet \\
\hline Substantia Nigra & Heart Left Ventricle & Hippocampus \\
Nucleus Accumbent Basal Ganglia & Amygdala & Whole Blood \\
Caudate Basal Ganglia & Hippocampus & Caudate Basal Ganglia \\
Putamen Basal Ganglia & Whole Blood & Substantia Nigra \\
Hippocampus & Putamen Basal Ganglia & Putamen Basal Ganglia \\
Amygdala & Substantia Nigra & Muscle Skeletal \\
Heart Left Ventricle & Anterior Cingulate Cortex & Nucleus Accumbent Basal Ganglia \\
Spinal Cord & Muscle Skeletal & Anterior Cingulate Cortex \\
Hypothalamus & Heart Atrial Appendage & Amygdala \\
Pancreas & Caudate Basal Ganglia & Brain Cortex \\
Heart Atrial Appendage & Nucleus Accumbent Basal Ganglia & Heart Left Ventricle \\
Anterior Cingulate Cortex & Brain Cortex & Kidney Medulla \\
Kidney Medulla & Hypothalamus & Frontal Cortex \\
\hline
\end{tabular}


Table A5. Overlapping genes between ataxias (dominant and recessive genes) and Parkinson's disease with the genes from the SFARI portal. Scores in parenthesis refer to the scoring of the gene according to the SFARI site (see Methods for explanation on each category). Syndromic is (4). Supplementary Figures S5-S18 provide the GTEx violin plots of these gene expressions in the top-ranked tissues unveiled by our analyses. Supplementary Table S2 compiles additional information on the genes from various sources in the clinical literature.

\begin{tabular}{ccc}
\hline $\begin{array}{c}\text { SFARI Autism and } \\
\text { Ataxia Dominant }\end{array}$ & $\begin{array}{c}\text { SFARI Autism and } \\
\text { Ataxia Recessive }\end{array}$ & $\begin{array}{c}\text { SFARI Autism and } \\
\text { (Early Onset) PD }\end{array}$ \\
\hline CACNA1C (1) & KCNJ10 (2) & RAB39B (3) \\
SCN2A (1) & WWOX (2) & \\
ATP1A3 (3S) & GRID2 (3) \\
CCDC88C (3) & LAMA1 (3) \\
ITPR1 (3) & PEX7 (3) & \\
& SYNE1 (3S) & \\
& CYP27A1 (4) \\
\end{tabular}

Table A6. Most-affected tissues upon removal of the DisGeNet genes associated with different types of ataxias, color coded by CNS (brain and spinal cord), heart-related, muscle-skeletal, and peripheral vital organ for systemic functioning. Predominance of CNS is evident, followed by heart-related and muscle-skeletal and peripheral organs.

\begin{tabular}{cccc}
\hline Gait Ataxia & Spinocerebellar Ataxia & Cerebellar Ataxia & Progressive-C Ataxia \\
\hline Heart Left Ventricle & Caudate Basal Ganglia & Heart Left Ventricle & Liver \\
Substantia Nigra & Brain Amygdala & Substantia Nigra & Heart Left Ventricle \\
Hippocampus & Substantia Nigra & Hippocampus & Whole Blood \\
Muscle Skeletal & Putamen Basal Ganglia & Amygdala & Heart Atrial Appendage \\
Caudate Basal Ganglia & N Accumbens BG & Whole Blood & Putamen Basal Ganglia \\
N Accumbens BG & Ant Cingulate Cortex & Putamen Basal Ganglia & Substantia Nigra \\
Whole Blood & Hippocampus & Hypothalamus & Hippocampus \\
Brain Amygdala & Heart Atrial Appendage & Ant Cingulate Cortex & Brain Amygdala \\
Kidney Cortex & Hypothalamus & Caudate Basal Ganglia & Muscle Skeletal \\
Putamen Basal Ganglia & Brain Cortex & N Accumbens BG & Kidney Cortex \\
Ant Cingulate Cortex & Frontal Cortex & Brain Cortex & Caudate Basal Ganglia \\
Hypothalamus & Heart Left Ventricle & Kidney Cortex & N Accumbens BG \\
Heart Atrial Appendage & Kidney Cortex & Muscle Skeletal & Ant Cingulate Cortex \\
\hline
\end{tabular}

Table A7. Most-affected tissues upon removal of the DisGeNet genes associated with schizophrenia (2697) from the human GTEx database; depression genes (1468); unipolar depression genes (641); and bipolar depression genes (116). Convergence between schizophrenia and depression is high, with maximally affected CNS tissues (10/13), followed by heart-related and muscle-skeletal tissues. Unipolar and bipolar depression also show systemic effect of vital peripheral organs ( $\mathrm{N}$ stands for Nucleus, Ant for Anterior, and BG for Basal Ganglia).

\begin{tabular}{cccc}
\hline Schizophrenia & Depression & Unipolar Depression & Bipolar Depression \\
\hline Amygdala & Heart Left Ventricle & Putamen Basal Ganglia & Hippocampus \\
Putamen Basal Ganglia & Heart Atrial Appendage & Caudate Basal Ganglia & Putamen Basal Ganglia \\
Heart Left Ventricle & Hippocampus & Hippocampus & Amygdala \\
Hippocampus & Muscle Skeletal & Substantia Nigra & Caudate Basal Ganglia \\
Substantia Nigra & Putamen Basal Ganglia & Kidney Cortex & Muscle Skeletal \\
Caudate Basal Ganglia & Caudate Basal Ganglia & Amygdala & Substantia Nigra \\
Ant Cingulate Cortex & Amygdala & Heart Left Ventricle & Spinal Cord \\
N Accumbens BG & Pancreas & Kidney Medulla & Esophagus Muscularis \\
Hypothalamus & Kidney Medulla & Brain Cortex & Adrenal Gland \\
Muscle Skeletal & Substantia Nigra & N Accumbens BG & Frontal Cortex \\
Pancreas & Kidney Cortex & Colon Transverse & Minor Salivary Gland \\
Brain Cortex & Ant Cingulate Cortex & Ant Cingulate Cortex & Colon Sigmoid \\
Frontal Cortex & N Accumbens BG & Heart Atrial Appendage & Ant Cingulate Cortex \\
\hline
\end{tabular}


Table A8. Most-affected tissues upon removal of the DisGeNet genes associated with colon cancer (3669) from the human GTEx database; irritable bowel syndrome genes (1483); congenital heart disease genes (267); and hematologic neoplast genes (827). Color code as in previous tables.

\begin{tabular}{c|ccc}
\hline Colon Cancer & $\begin{array}{c}\text { Irritable Bowel } \\
\text { Syndrome }\end{array}$ & $\begin{array}{c}\text { Congenital Heart } \\
\text { Disease }\end{array}$ & Hematologic Neoplast \\
\hline Pancreas & Heart Left Ventricle & Heart Left Ventricle & Heart Left Ventricle \\
Whole Blood & Pancreas & Heart Atrial Appendage & Whole Blood \\
Stomach & Kidney Cortex & Kidney Cortex & Liver \\
Adrenal Gland & Liver & Whole Blood & Heart Atrial Appendage \\
Colon Transverse & Muscle Skeletal & Kidney Medulla & Pancreas \\
Prostate & Heart Atrial Appendage & Substantia Nigra & Muscle Skeletal \\
Small Intestine T & Kidney Medulla & Hypothalamus & Kidney Cortex \\
Spleen & Whole Blood & Prostate & Hippocampus \\
Minor Salivary Gland & Adrenal Gland & Hippocampus & Kidney Medulla \\
Fallopian Tube & Adipose Viseral Oment & Muscle Skeletal & Esophagus Mucosa \\
EBT Lymphocytes & Esophagus Mucosa & Adipose Visceral O & EBT Lymphocytes \\
SkinNoSunExposed S & Colon Transverse & Minor Salivary Gland & Substantia Nigra \\
Liver & Stomach & Pancreas & Putamen Basal Ganglia \\
\hline
\end{tabular}

Table A9. Most-affected tissues upon removal of the DisGeNet genes associated with lupus systemic erythematosus (1883) from the human GTEx database; psoriasis genes (1308); breast cancer (510); and diabetes genes (3134). The top half of the highest-ranked tissues show no convergence with CNS-related tissues found in the mental illnesses and neurological disorders interrogated in this work. Instead, heart-related tissue, muscle-skeletal tissue and tissues related to peripheral vital organs for systemic functioning are found. The bottom half of the top $\Delta \lambda$-ranked tissues are a mixture of tissues in peripheral bodily organs and brain-related tissues. The latter are from motor control, coordination, and adaptation subcortical areas and from emotion, memory, and regulatory areas. Color code as in previous tables.

\begin{tabular}{|c|c|c|c|}
\hline $\begin{array}{l}\text { Systemic Lupus } \\
\text { Erythematosus }\end{array}$ & Psoriasis & Breast Cancer & Diabetes \\
\hline Heart Left Ventricle & Heart Left Ventricle & Pancreas & Liver \\
\hline Whole Blood & Liver & Heart Left Ventricle & Pancreas \\
\hline Liver & Whole Blood & Heart Atrial Appendage & Heart Atrial Appendage \\
\hline Heart Atrial Appendage & Muscle Skeletal & Kidney Medulla & Heart Left Ventricle \\
\hline Pancreas & Heart Atrial Appendage & Muscle Skeletal & Kidney Medulla \\
\hline Muscle Skeletal & Pancreas & Whole Blood & Kidney Cortex \\
\hline Kidney Cortex & Kidney Cortex & Ant Cingulate Cortex & Putamen Basal Ganglia \\
\hline Hippocampus & Putamen Basal Ganglia & Esophagus Mucosa & Substantia Nigra \\
\hline Kidney Medulla & Caudate Basal Ganglia & Kidney Cortex & Hippocampus \\
\hline Esophagus Mucosa & Adipose Visceral O & Adrenal Gland & Caudate Basal Ganglia \\
\hline EBT Lymphocytes & Substantia Nigra & Esophagus Muscularis & Amygdala \\
\hline Substantia Nigra & Esophagus Mucosa & Hypothalamus & N Accumbens BG \\
\hline Putamen Basal Ganglia & Hypothalamus & Putamen Basal Ganglia & Hypothalamus \\
\hline
\end{tabular}


Table A10. Most-affected tissues upon removal of the DisGeNet genes associated with mitochondrial disease (284) from the human GTEx database; mitochondrial myopathies genes (121); mitochondrial encephalopathy, lactic acidosis, and stroke-like episodes (MELAS) (81); Post-Traumatic Stress Disorder genes (418).

\begin{tabular}{cccc}
\hline Mitochondrial Disease & $\begin{array}{c}\text { Mitochondrial } \\
\text { Myopathies }\end{array}$ & MELAS & PTSD \\
\hline Heart Left Ventricle & Liver & Heart Left Ventricle & Caudate Basal Ganglia \\
Muscle Skeletal & Heart Left Ventricle & Liver & Kidney Medulla \\
Kidney Cortex
\end{tabular}

Table A11. Most-affected tissue in each disease/disorder according to the maximum $\Delta \lambda$ value (See Figure 13 in the main text).

\begin{tabular}{cc}
\hline Disease/Disorder & Maximally Affected Tissue \\
\hline Autism DisGeNet & BrainSubstantiaNigra \\
ASD NoAtaxias & BrainPutamenBasalGanglia \\
Autism SFARI & BrainPutamenBasalGanglia \\
Schizophrenia & BrainAmygdala \\
Depression DisGeNet & HeartLeftVentricle \\
Bipolar Depression & BrainHippocampus \\
Unipolar Depression & BrainPutamenBasalGanglia \\
ATAXIA DisGeNet & HeartLeftVentricle \\
SpinoCerebellar ATAXIA & BrainCaudateBasalGanglia \\
Cerebellar ATAXIA & HeartLeftVentricle \\
ProgressiveCerebellar ATAXIA & Liver \\
Gait ATAXIA & HeartLeftVentricle \\
FAXTAS & HeartLeftVentricle \\
FX & BrainHippocampus \\
PTSD & BrainCaudateBasalGanglia \\
Diabetes & Liver \\
BreastCancer & Pancreas \\
ColonCancer & Pancreas \\
HematologicNeoplast & HeartLeftVentricle \\
CongenitalHeartDisease & HeartLeftVentricle \\
IBD & HeartLeftVentricle \\
LupusSystemicErythomatosus & HeartLeftVentricle \\
Psoriasis & HeartLeftVentricle \\
MItochondriaDisease & HeartLeftVentricle \\
MELAS & HeartLeftVentricle \\
MitochondriaMyopathies & Liver \\
\hline
\end{tabular}

\section{References}

1. Hawgood, S.; Hook-Barnard, I.G.; O’Brien, T.C.; Yamamoto, K.R. Precision medicine: Beyond the inflection point. Sci. Transl. Med. 2015, 7, 300ps17. [CrossRef]

2. Adams, R.A.; Huys, Q.J.M.; Roiser, J.P. Computational psychiatry: Towards a mathematically informed understanding of mental illness. J. Neurol. Neurosurg. Psychiatry 2016, 87, 53-63. [CrossRef] 
3. American Psychiatric Association. Diagnostic and Statistical Manual of Mental Disorders, 5th ed.; American Psychiatric Association: Arlington, VA, USA, 2013.

4. Ferraro, N.M.; Strober, B.J.; Einson, J.; Abell, N.S.; Aguet, F.; Barbeira, A.N.; Brandt, M.; Bucan, M.; Castel, S.E.; Davis, J.R.; et al. Transcriptomic signatures across human tissues identify functional rare genetic variation. Sci. Mag. 2020, 369, eaaz5900.

5. Torres, E.; Denisova, K. Motor noise is rich signal in autism research and pharmacological treatments. Sci. Rep. 2016, 6, 37422. [CrossRef]

6. Torres, E.; Caballero, C.; Mistry, S. Aging with Autism Departs Greatly from Typical Aging. Sensors 2020, 20, 572. [CrossRef] [PubMed]

7. Caballero, C.; Mistry, S.; Torres, E. Age-dependent statistical changes of involuntary head motion signatures across autism and controls of the ABIDE repository. Front. Integr. Neurosci. 2020, 14, 1-14. [CrossRef] [PubMed]

8. Kim-Hellmuth, S.; Aguet, F.; Oliva, M.; Muñoz-Aguirre, M.; Kasela, S.; Wucher, V.; Castel, S.E.; Hamel, A.R.; Viñuela, A.; Roberts, A.L.; et al. Cell type-specific genetic regulation of gene expression across human tissues. Sci. Mag. 2020, 369, eaaz8528. [CrossRef] [PubMed]

9. Nguyen, J.; Majmudar, U.; Papathomas, T.V.; Silverstein, S.; Torres, E.; Thomas, V.P. Schizophrenia: The micro-movements perspective. Neuropsychologia 2016, 85, 310-326. [CrossRef]

10. Rogers, D.M. Motor Disorder in Psychiatry: Towards A Neurological Psychiatry; J. Wiley \& Sons.: Chichester, UK; New York, NY, USA, 1992; Volume viii, p. 159.

11. Bird, T.D. Hereditary Ataxia Overview, in GeneReviews((R)); Adam, M., Ardinger, H.H., Pagon, R.A., Wallace, S.E., Bean, L.J.H., Stephens, K., Amemiya, A., Eds.; University of Washington: Seattle, WA, USA, 1998.

12. Jayadev, S.; Bird, T.D. Hereditary ataxias: Overview. Genet. Med. 2013, 15, 673-683. [CrossRef]

13. Chinnery, P.F. Mitochondrial Disorders Overview, in GeneReviews((R)); University of Washington: Seattle: Seattle, WA, USA, 1993.

14. Domingo, A.; Westenberger, A.; Lee, L.V.; Brænne, I.; Liu, T.; Vater, I.; Rosales, R.; Jamora, R.D.G.; Pasco, P.M.; Paz, E.M.C.-D.; et al. New insights into the genetics of X-linked dystonia-parkinsonism (XDP, DYT3). Eur. J. Hum. Genet. 2015, 23, 1334-1340. [CrossRef]

15. Klein, C.; Westenberger, A. Genetics of Parkinson's disease. Cold Spring Harb. Perspect. Med. 2012, 2, a008888. [CrossRef]

16. Scott, W.K.; Nance, M.A.; Watts, R.L.; Hubble, J.P.; Koller, W.C.; Lyons, K.; Pahwa, R.; Stern, M.B.; Colcher, A.; Hiner, B.C.; et al. Complete genomic screen in Parkinson disease: Evidence for multiple genes. JAMA 2001, 286, 2239-2244. [CrossRef]

17. Hicks, A.; Petursson, H.; Jonsson, T.; Stefansson, H.; Jóhannsdóttir, H.S.; Sainz, J.; Frigge, M.L.; Kong, A.; Gulcher, J.; Stefansson, K.; et al. A susceptibility gene for late-onset idiopathic Parkinson's disease. Ann. Neurol. 2002, 52, 549-555. [CrossRef] [PubMed]

18. Valente, E.M.; Brancati, F.; Ferraris, A.; Bsc, E.A.G.; Davis, M.B.; Breteler, M.M.; Gasser, T.; Bonifati, V.; Bentivoglio, A.R.; de Michele, G.; et al. PARK6-linked parkinsonism occurs in several European families. Ann. Neurol. 2002, 51, 14-18. [CrossRef]

19. Kay, D.M.; Zabetian, C.P.; Factor, S.A.; Nutt, J.G.; Samii, A.; Griffith, A.; Bird, T.D.; Kramer, P.; Higgins, D.S.; Payami, H. Parkinson's disease and LRRK2: Frequency of a common mutation in U.S. movement disorder clinics. Mov. Disord. 2006, 21, 519-523. [CrossRef] [PubMed]

20. Soong, B.W.; Morrison, P.J. Spinocerebellar ataxias. Handb. Clin. Neurol. 2018, 155, 143-174.

21. Fujioka, S.; Sundal, C.; Wszolek, Z.K. Autosomal dominant cerebellar ataxia type III: A review of the phenotypic and genotypic characteristics. Orphanet J. Rare Dis. 2013, 8, 14. [CrossRef] [PubMed]

22. Sailer, A.; Houlden, H. Recent advances in the genetics of cerebellar ataxias. Curr. Neurol. Neurosci. Rep. 2012, 12, 227-236. [CrossRef]

23. Mariotti, C.; di Donato, S. Cerebellar/spinocerebellar syndromes. Neurol. Sci. 2001, 22 (Suppl. 2), S88-S92. [CrossRef]

24. Mosconi, M.W.; Wang, Z.; Schmitt, L.M.; Tsai, P.T.; Sweeney, J.A. The role of cerebellar circuitry alterations in the pathophysiology of autism spectrum disorders. Front. Neurosci. 2015, 9, 296. [CrossRef]

25. Mostofsky, S.H.; Powell, S.K.; Simmonds, D.J.; Goldberg, M.C.; Caffo, B.; Pekar, J.J. Decreased connectivity and cerebellar activity in autism during motor task performance. Brain 2009, 132, 2413-2425. [CrossRef] [PubMed] 
26. Ryu, J.; Vero, J.; Dobkin, R.D.; Torres, E.B. Dynamic digital biomarkers of motor and cognitive function in parkinson's disease. J. Vis. Exp. 2019, 149, e59827. [CrossRef] [PubMed]

27. Wu, D.; José, J.V.; Nurnberger, J.I.; Torres, E. A Biomarker Characterizing Neurodevelopment with applications in Autism. Sci. Rep. 2018, 8, 614. [CrossRef]

28. Torres, E.; Vero, J.; Rai, R. statistical platform for individualized behavioral analyses using biophysical micro-movement spikes. Sensors 2018, 18, 1025. [CrossRef] [PubMed]

29. Torres, E.; Brincker, M.; Isenhower, R.W.I.; Yanovich, P.; Stigler, K.A.; Nurnberger, J.I.; Metaxas, D.N.; José, J.V. Autism: The micro-movement perspective. Front. Integr. Neurosci. 2013, 7, 32. [CrossRef]

30. Salleh, M.R. The genetics of schizophrenia. Malays. J. Med. Sci. 2004, 11, 3-11.

31. Schizophrenia Working Group of the Psychiatric Genomics Consortium; Ripke, S.; Neale, B.M.; Corvin, A.; Walters, J.; Farh, K.-H.; Holmans, P.; Lee, P.; Bulik-Sullivan, B.; Collier, D.A.; et al. Biological insights from 108 schizophrenia-associated genetic loci. Nature 2014, 511, 421-427.

32. Kotlar, A.V.; Mercer, K.B.; Zwick, M.E.; Mulle, J.G. New discoveries in schizophrenia genetics reveal neurobiological pathways: A review of recent findings. Eur. J. Med. Genet. 2015, 58, 704-714. [CrossRef]

33. Fabbri, C.; Serretti, A. Role of 108 schizophrenia-associated loci in modulating psychopathological dimensions in schizophrenia and bipolar disorder. Am. J. Med. Genet. B Neuropsychiatr. Genet. 2017, 174, 757-764. [CrossRef]

34. Forsyth, J.K.; Asarnow, R.F. Genetics of childhood-onset schizophrenia 2019 update. Child Adolesc. Psychiatr. Clin. N. Am. 2020, 29, 157-170. [CrossRef]

35. Zamanpoor, M. Schizophrenia in a genomic era: A review from the pathogenesis, genetic and environmental etiology to diagnosis and treatment insights. Psychiatr. Genet. 2020, 30, 1-9. [CrossRef] [PubMed]

(C) 2020 by the author. Licensee MDPI, Basel, Switzerland. This article is an open access article distributed under the terms and conditions of the Creative Commons Attribution (CC BY) license (http://creativecommons.org/licenses/by/4.0/). 